\title{
Lebende Tiere und inszenierte Natur
}

\author{
Zeichnung und Fotografie in der populären Zoologie zwischen 1860 \\ und 1910
}

\author{
Alexander Gall
}

Live Animals and Staged Nature. Drawing and Photography in German Popular Zoology between 1860 and 1910

\begin{abstract}
It is the central thesis of this paper that the "biological perspective" (Lynn Nyhart) typical for Germany, with its interest in living animals, not only influenced natural history practices in many ways during the second half of the 19th century, rather also shaped the illustrations of popular zoology publications, as for example those in Brehms Thierleben. The illustrators of this period preferred to use live animals as models, which they studied in zoos. These animals were often depicted in their "natural" habitats. Since the illustrators knew only very little about these habitats, they had to be imagined. Another fashionable genre within popular zoology was the portrayal of animals fighting, which attracted attention because of their drama. The first wildlife photographers oriented themselves on the zoological illustrations and, with the aid of manipulation, staging and retouching, gave their photographs the impression of natural surroundings and drama. Yet both the illustrators and the photographers emphasized their truth to nature and - based on this - the scientific value of their pictures. In so doing, they developed a "biological" kind of wildlife photography, which, after the turn of the 19th century, allowed dedicated amateurs to create a popular zoological oeuvre that was well received by broad audiences.
\end{abstract}

Keywords: Animal illustration, Wildlife photography, Biology, Popular science, Natural history

In dem Beitrag wird die These vertreten, dass die für Deutschland typische „biologische Perspektive" (Lynn Nyhart) mit ihrem Interesse am lebenden Tier nicht nur die naturkundliche Praxis während der zweiten Hälfte des 19. Jahrhunderts in vielfältiger Weise beeinflusste, sondern auch die Illustrationen der populären Zoologie, wie etwa in Brehms Thierleben, prägte: Die Zeichner bevorzugten als Modelle nun lebende Tiere, die sie in den zoologischen Gärten studierten; dabei stellten sie diese häufig in ihrer natürlichen Umgebung dar, die sie aus Mangel an eigener Kenntnis aber imaginieren mussten. Ein weiteres beliebtes Genre in der populären Zoologie waren die Darstellungen von kämpfenden Tieren, die nicht zuletzt durch ihre Dramatik ins Auge fielen. Die ersten Tierfotografen orientierten sich an diesen Merkmalen der zoologischen Illustrationen und gaben ihren Aufnahmen mit Hilfe von Manipulationen, Inszenierungen und Retuschen den Eindruck von natürlicher Umgebung und Dramatik. Dennoch betonten sowohl Zeichner als auch Fotografen die Naturtreue und - darauf beruhend - den wissenschaftlichen Wert ihrer Abbildungen. Sie prägten damit eine „biologische" Tierfotografie, die es nach der Jahrhundertwende engagierten Amateuren ermöglichte ein populäres Werk der heimischen Tierwelt zu schaffen, das breite Anerkennung fand.

Schlüsse/wörter: Tierzeichnung, Tierfotografie, Biologie, Populärwissenschaft, Naturkunde

Als der Berliner Werner Verlag 1899 unter dem Titel Lebende Bilder aus dem Reiche der Tiere eines der ersten mit Fotografien illustrierten Tierbücher in Deutschland veröffentlichte, hieß es in einer Rezension, dass das 
Werk „wirkliches Leben“ darbiete, „thierisches Leben in einer Vollkraft, nahe dem der Freiheit und Wildniss [sic!]“. Es bringe „zum ersten Male zum Bewusstsein, von welch' unendlichem Werthe das photographische Augenblicks-Bild des Thieres nicht nur für das Studium des Künstlers, sondern auch für die Förderung des allgemeinen Wissens" sei (Anonym 1899: 240, 242). Aus diesen Zeilen spricht zum einen die große Bedeutung, die die Darstellung „lebender“ Tiere besaß, zum anderen kommt die Begeisterung über die neuen durch die Fotografie verfügbaren Möglichkeiten zum Ausdruck und dann lässt sich noch die breite gesellschaftliche Reichweite der populären Zoologie in Deutschland ablesen, denn die Besprechung war in einer Kunst- und nicht etwa in einer Naturkundezeitschrift erschienen.

"Populäre Zoologie“ meint hier den Diskurs zoologischer Themen in den verschiedenen Sphären der Öffentlichkeit - außerhalb universitärer und wissenschaftlicher Fachkreise - von stark interessierten und teilweise auch engagierten Personen bis hin zum Gelegenheitsleser entsprechender Berichte in den zeitgenössischen Massenmedien. Eine Rückkehr zum Defizitmodell populären Wissens, das der Begriff der Wissenschaftspopularisierung häufig impliziert, ist damit nicht verbunden, Wissenschaftsvermittlung wird vielmehr als Prozess mit eigener Logik und Rückwirkungen auf die Fachwissenschaft verstanden (Schirrmacher 2008: 79-88). Für die Öffentlichkeit übernahm die Biologie und insbesondere die Zoologie während der zweiten Hälfte des 19. Jahrhunderts als Wissenschaft eine Leitfunktion, die sich auch in einer Spitzenposition auf dem populärwissenschaftlichen Buchmarkt bemerkbar machte. Als Gründe für dieses öffentliche Interesse werden neben der Bedeutung des Darwinismus die Möglichkeiten zu amateurwissenschaftlicher Betätigung, ihre ästhetisch-sinnliche Wirkung, ihre Anschaulichkeit und korrespondierend dazu die reichen Illustrationen populärer zoologischer Publikationen angeführt (Daum 2002: $431 \mathrm{f}$.; Engels 1995; Schwarz 1999: 117, 2007: 65).

Aber anders als die wissenschaftlichen Abbildungen der Biologie (z. B. Chadarevian 1994; Hopwood 2005; Voss 2007; Wittmann 2008) haben zoologische Illustrationen in populären Medien wie Familienzeitschriften und Sachbüchern trotz des iconic turn bisher nur wenig Aufmerksamkeit erfahren. ${ }^{1}$ Außerdem wurde es bisher versäumt, die aufkommende Fotografie in die Geschichte der populären Zoologie zu integrieren und zur Tierzeichnung in Beziehung zu setzen. Stärkere Beachtung in der Forschung fand bisher nur der kleine Ausschnitt von Eadweard Muybridges und Etienne-Jules Mareys chronofotografischen Tieraufnahmen (Braun 1992, 2010; Clegg 2007). Zum Gros der Tierfotografien existieren dagegen nur wenige Arbeiten, die entweder Ereignisgeschichte und Aufnahmetechnik behandeln (Guggisberg 1977; Storsberg 2010: 33-165) oder die Verbindungen zu Jagd, Männlichkeit, Kolonialismus und Naturschutz herausarbeiten 
(Ryan 1997: 99-139; Dunaway 2000; Gissibl 2005; Brower 2010: 25-82; Voss 2015).

Im Folgenden wird nun die enge Verbindung von populärer Tierzeichnung und früher Tierfotografie aufgezeigt und die These vertreten, dass beide Verfahren die gleichen visuellen Strategien verfolgten, obwohl sie sich dem ersten Eindruck nach in ihrer Darstellungsweise deutlich voneinander unterscheiden. Sowohl die Zeichner beziehungsweise ihre Auftraggeber als auch die Fotografen teilten nämlich, so der zweite Teil dieser These, vielfach eine Sicht auf Natur und Tierwelt, die durch das Interesse am lebenden Tier, an seinem Lebensraum und seiner Lebensweise bestimmt war. In der historischen Rückschau wurde diese Sicht von Lynn Nyhart (2009) als „biologische Perspektive“ bezeichnet, die zwar nicht als Konzept ausformuliert war, aber mit ihren Vorstellungen, Betrachtungsweisen und den ihr zugrundliegenden Dispositionen während der zweiten Hälfte des 19. Jahrhunderts die Arbeit vieler praktisch tätiger Naturkundler in Deutschland prägte. Dieser Kreis von „Praktikern der Naturgeschichte“ setzte sich unter anderem aus Jägern und Tierfängern zusammen, die für Museen, Zoos und Menagerien arbeiteten; er umfasste Tierpräparatoren und Konservatoren in Museen sowie Direktoren und Inspektoren der neu gegründeten Tiergärten und schließlich auch Wissenschaftsvermittler und Naturschützer, die sich in Vorträgen, Artikeln und Büchern an ein breites Publikum wandten. Als ein zentrales Forum diente ihnen die 1859 gegründete Zeitschrift Der Zoologische Garten, die in den folgenden Jahrzehnten auch vielen naturkundlich interessierten Amateuren die Möglichkeit gab, an einem wissenschaftlichen Diskurs zu partizipieren. Die „biologische Perspektive“ definiert sich deshalb auch durch die Zugehörigkeit zu diesen Gruppen und in Abgrenzung zur universitären Zoologie, die sich primär Fragen der Systematik widmete und kaum zur „Biologie“ der Wirbeltiere forschte (Hochadel 2011; Nyhart 2009: 4-6, 22-24, 35-38, 80 f.; Rieke-Müller \& Dittrich 1998: 30-39). Gegen Ende des Jahrhunderts verlor dieser Gegensatz aber an Bedeutung, da biologische Themen inzwischen Eingang in die akademische Forschung gefunden hatten.

Einen wichtigen, von Nyhart meiner Meinung nach vernachlässigten, Einfluss auf die „biologische Perspektive“ übte die romantische Naturphilosophie aus, zu deren deutschsprachigen Vertretern unter anderen der Mediziner Lorenz Oken (1779-1851), der Pfarrer, Philosoph und Naturkundler Peter Scheitlin (1779-1848) sowie die Ornithologen Johann Friedrich Naumann (1780-1857) und Christian Ludwig Brehm (1787-1864) zählten. Außerhalb der universitären Zoologie verbreiteten sie den Glauben an eine den höheren Tieren eigene Seele und ein anthropomorphes Tierverständnis, das Alfred Edmund Brehm (1829-1884) dann mit einem hohen $\mathrm{Ma}$ an rhetorischer Gestaltung auf die Spitze trieb (Haemmerlein 
1989: 22-24; Haffer 2001: 35 f.; Daum 2002: 258). Zu den Anhängern der „Tierseelenkunde“ gehörte auch Brehms Freund David Friedrich Weinland (1829-1915), der als Sekretär der Zoologischen Gesellschaft in Frankfurt am Main die bereits erwähnte Zeitschrift Der Zoologische Garten gründete und vier Jahre lang herausgab (Weinland 1860a; Rieke-Müller 1995: 483). Das Interesse an der Tierseele ging Hand in Hand mit der „biologischen Perspektive“, da es nahezu allen tierischen Lebensäußerungen Relevanz verlieh.

Die Darstellung von Tieren war von der „biologischen Perspektive“ auf vielfältige Weise betroffen, wie Nyhart (2009: 251-292) am Beispiel von deren Präparation und musealen Präsentation in Form „biologischer Gruppen" aufgezeigt hat. Um nun die Auswirkungen auf die Tierzeichnung $\mathrm{zu}$ untersuchen, bietet sich aus mehreren Gründen Brehms Thierleben an: Denn es erreichte nicht nur hohe Auflagenzahlen und eine breite Leserschaft (Jäger 2001: $566 \mathrm{f}$.), sondern wurde mit seinen Illustrationen auch rasch zum Vorbild für die populäre Tierzeichnung (Artinger 1995: 13f.). Außerdem gehörte Brehm zur ersten Generation von Naturkundlern, denen Nyhart (2009: 36-45) eine „biologische Perspektive“ zuschreibt. Hohe Auflagenzahlen und eine breite Leserschaft kennzeichneten auch die Familienzeitschrift Gartenlaube. In die Analyse wurde sie vor allem als Beispiel für die Dramatisierung zoologischer Illustrationen einbezogen, die in populären Tierkundebüchern eher die Ausnahme blieb (Gall 2011: 111-119). Diese Auswahl repräsentiert also nicht das gesamte Spektrum der populären Tierzeichnung, sondern konzentriert sich auf die Beispiele, die besonders einflussreich und/oder weit verbreitet waren. Dagegen fanden für die Tierfotografie nicht nur die Aufnahmen des bekannten Fotopioniers Ottomar Anschütz (1846-1907) Beachtung, sondern, nach meinem Kenntnisstand, alle weiter verbreiteten fotografisch illustrierten Buchpublikationen zur populären Säugetier- und Vogelkunde bis zur Veröffentlichung von Hermann Meerwarths Lebensbildern aus der Tierwelt im Jahr 1908, die fast zeitgleich erschienene Heftreihe Natur-Urkunden (1908/1909) von Georg Schulz (1875-1955) jedoch nur ganz am Rande. ${ }^{2}$

So wie zahlreiche Illustratoren und visuell arbeitende Wissenschaftler vor ihnen nahmen auch die Zeichner und Fotografen der populären Tierbilder seit der Mitte des 19. Jahrhunderts für ihre Aufzeichnungsverfahren Naturtreue in Anspruch; nur hatte ihr Verständnis davon nicht mehr viel mit der auf Typisierung abzielenden "Naturwahrheit" des 18. und frühen 19. Jahrhunderts gemeinsam (Daston \& Galison 2007: 70 f.), sondern war von der Konfrontation mit dem lebenden Tier geprägt. Trotz des gemeinsamen Anspruches auf Naturtreue distanzierten sich die Tierfotografen aber schon früh von der Zeichnung und kritisierten deren „Subjektivität“, um im Gegenzug die „Objektivität“ ihrer Aufnahmen hervorzuheben. 
Zugleich griffen sie aber immer wieder mit erheblichem Aufwand in den fotografischen Prozess ein, um ihren Bildern bestimmte Charakteristika der Zeichnungen zu verleihen. Schon zuvor hatten sich die Tierzeichner ganz ähnlich verhalten und die eigenen Ansprüche an Naturtreue zumindest teilweise unterlaufen, um bestimmten Vorstellungen von Auftraggebern und Publikum zu entsprechen. Sowohl für die Tierzeichnungen als auch für die Tierfotografien ergaben sich zwischen den Ansprüchen auf Naturtreue und Objektivität einerseits und den vorgenommenen Manipulationen andererseits also auffällige Diskrepanzen. Im Folgenden dient dies zum einen als zusätzliches Argument dafür, dass die Abbildungen durch die „biologische Perspektive“ geprägt waren. Zum anderen lassen sich diese Widersprüche als Chance nutzen, um die gängigen Vorstellungen von einer attraktiven Illustration oder einer gelungenen Aufnahme zu rekonstruieren und so auch die Erwartungen der Leserschaft ein Stück weit zu ergründen.

\section{Leben und imaginierte Natur in den Illustrationen von Brehms Thierleben}

An Alfred Edmund Brehms Leben lässt sich im folgenden Abschnitt exemplarisch vorführen, wie verschiedene naturkundliche Praktiken mit der „biologischen Perspektive“ verknüpft waren und was das für die Illustrationen seines Thierlebens bedeutete. Brehms Vater Christian Ludwig bekleidete eine Pastorenstelle im thüringischen Renthendorf, war aber zugleich einer der bekanntesten Ornithologen seiner Zeit und besaß eine umfangreiche Sammlung von Vogelbälgen. Er lehrte seinen Sohn den Umgang mit dem Jagdgewehr, das Sezieren, Präparieren und Bestimmen von Vögeln sowie die Kunst der Naturbeobachtung und vermittelte ihm damit zentrale Fähigkeiten naturkundlicher Praxis. Sie qualifizierten den gerade 18 Jahre alten Alfred als Begleiter einer Reise ins nordöstliche Afrika, die die Sammlung von Tieren zum Ziel hatte. Zum Ertrag der fünfährigen Afrikaexpedition (1847-1852) gehörten nicht nur eine Vielzahl von Vogelbälgen, einige fachwissenschaftliche Publikationen sowie seine populären Reiseskizzen (Brehm 1855), sondern vor allem Erfahrungen aus erster Hand mit lebenden exotischen Tieren (Rieke-Müller 1995: 479). Im Anschluss studierte er in Jena Zoologie und promovierte nach zwei Jahren mit einigen Kapiteln seiner Reiseskizzen. Weitere Forschungsreisen führten ihn 1856/57 nach Spanien und 1860 nach Norwegen, wo Brehm jeweils eine ihm bis dato unvertraute Vogelfauna studieren konnte. 
1858, nach seiner Rückkehr aus Spanien, zog Brehm nach Leipzig, wo er bald mit dem demokratischen Politiker und Wissenschaftsvermittler Emil Adolf Roßmäßler (1806-1867) Bekanntschaft schloss sowie mit Ernst Keil (1816-1878), dem Gründer der Familienzeitschrift Gartenlaube. Noch im selben Jahr gewann dieser Brehm als Autor und finanzierte ihm später die erwähnte Forschungsreise nach Norwegen, von der Brehm in mehreren Beiträgen für die Gartenlaube berichtete. Im Oktober 1860 schloss Brehm mit dem Verleger Herrmann Julius Meyer (1826-1909) den Vertrag für das Thierleben, das zu Beginn jedoch nur als Werk über die Säugetiere konzipiert war (Haemmerlein 2015: 66 f.). Die Arbeit an seinem ebenfalls populär gehaltenen Buch über das Leben der Vögel (Brehm 1861b) hatte Brehm zu diesem Zeitpunkt schon fast beendet. Im Frühjahr 1862 nahm er zunächst als Leiter und Organisator an einer Jagdexpedition Herzog Ernst II. von Sachsen-Coburg-Gotha nach Abessinien (heute Äthiopien) teil und zog dann nach Hamburg, um als Gründungsdirektor den neu geschaffenen Zoologischen Garten der Stadt aufzubauen.

Als typischer Praktiker der Naturgeschichte erwies sich Brehm in seiner zunehmenden Kritik an der universitären Zoologie, der er ein Desinteresse am lebenden Tier vorwarf. Solange er sich wie sein Vater primär mit ornithologischen Themen beschäftigt hatte, hatte dazu kaum Anlass bestanden. Denn sowohl die deutsche Ornithologen-Gesellschaft als auch das Journal für Ornithologie behandelten ein breites Spektrum von Fragen, zu denen auch die Lebensweise der Vögel gehörte (Rieke-Müller \& Dittrich 1998: $31 \mathrm{f}$.$) .$

Als Brehm sich durch die Arbeit am Thierleben verstärkt für die Säugetiere zu interessieren begann, bemerkte er schon in seinem Bericht über die Abessinienreise, dass „in den neueren thierkundlichen Werken [...] sonderbarer Weise das Leben der Thiere kaum berücksichtigt" werde. „Man begnügt sich mit genauen Beschreibungen des Leibes und wendet weitaus die größte Aufmerksamkeit auf die Zergliederung desselben [...], während über die Lebensweise, die Sitten, Gewohnheiten, die Nahrung u.s.w. meist ein tiefes Stillschweigen herrscht" (Brehm 1863a: 71, Hervorh. i. Orig.). In dem Anfang Januar 1865 geschriebenen Vorwort zum Illustrirten Thierleben spitzte er diese Kritik weiter zu: Während es für die „Meister der Thierkunde“ offenbar „unvereinbar mit der Wissenschaftlichkeit“ zu sein scheint, dem „Leben und Treiben der Thiere mehr Zeit und Raum zu gönnen“ als unbedingt nötig, sind es nur die sonst als „Gehilfen und Handlanger“ angesehenen ,jagenden und sammelnden Reisenden“, die „uns mit dem ganzen Thiere bekannt machen“: „Denn erst das lebende Thier ist ein „fühlendes und bewegungsfähiges“ Wesen: das todte, ausgestopfte und in Weingeist aufbewahrte ist und bleibt immer nur ein Gegenstand." Dagegen 
wollte Brehm ein Werk schaffen, „welches dem Leben sein Recht werden ließe" (Brehm 1865a: VII-VIII, Hervorh. i. Orig.; Rieke-Müller 1995: 480).

Einen passenderen Titel als Thierleben hätten er beziehungsweise sein Verlag deshalb wohl kaum finden können; eine Neuschöpfung war er aber nicht, sondern unter anderem bereits 1853 von Friedrich von Tschudi (1820-1886) für sein Thierleben der Alpenwelt verwendet worden (Haemmerlein 2015: $69 \mathrm{f}$.). Trotzdem besaß er noch ein gewisses Maß an Originalität, denn die gängige zeitgenössische Bezeichnung für vergleichbare Werke lautete „Naturgeschichte“. Dass „dieses gute deutsche Wort“ nicht als Titel diente, war tatsächlich der einzige Kritikpunkt einer ansonsten überaus wohlwollenden Besprechung der ersten drei Heftlieferungen im Zoologischen Garten (Anonym 1863a).

Doch inwieweit galt diese am lebenden Tier orientierte Programmatik auch für die Illustrationen? Um diese Frage zu beantworten, muss man sich zunächst die Situation naturkundlicher Zeichner und die Praxis der zoologischen Zeichnung in der ersten Hälfte des 19. Jahrhunderts vergegenwärtigen. Bis in seine Anfangsjahre galt die enge Zusammenarbeit von Zeichner und Naturforscher als wesentliche Voraussetzung für gelungene wissenschaftliche Abbildungen (Daston \& Galison 2007: 88-103). Zumindest für Deutschland lässt sich für die Zeit danach die Tendenz ausmachen, dass die zoologischen Illustratoren ihre ästhetischen Ansprüche hinter die Forderungen der Naturwissenschaftler zurückstellten und damit an Eigenständigkeit verloren. Ihre Tätigkeit wurde als dienend abqualifiziert und mit der von Handwerkern gleichgesetzt, so dass ihr Prestige als Künstler schwand (Ludwig 2000: 86; Schulze 2005: 153). Grundsätzlich stützten sich die Zeichner für ihre Arbeit stark auf vorhandene Abbildungen, die sie kopierten, um das vorhandene Wissen optimal auszuschöpfen und bei Ergänzungen oder der Korrektur von Details die Fehler eines vollständig neuen Entwurfs zu vermeiden. ${ }^{3}$ Als Vorlage nutzten sie außerdem Präparate, in der Botanik also getrocknete, in Herbarien gesammelte Pflanzenbelege und in der Zoologie ausgestopfte Tierhäute. Zusammen mit dem entfernten und getrennt präparierten Skelett dienten sie zur Klärung taxonomischer Fragen, die damals im Zentrum der wissenschaftlichen Arbeit standen; zugleich lieferten sie die Vorlagen für Illustrationen, die Tiere nicht in ihrer Individualität, sondern als typische Exemplare ihrer Art und meist von ihrer Umgebung isoliert zeigten (Blum 1993: 48).

Wenn Zeichnungen jedoch an ein breiteres Publikum adressiert waren, dann stellten die meist statisch montierten Bälge sowie Skelette, Felle und andere verwahrte Überreste die Zeichner nicht nur vor die Aufgabe, daraus wieder einen vollständigen Organismus zusammenzufügen, sondern diesem auch neues Leben einzuhauchen (Voss 2005: 234). Doch ohne die Kenntnis des lebenden Tieres war die Gefahr groß, nur die Statik und Leb- 
losigkeit der Präparate in der Zeichnung zu reproduzieren. Zahllose Beispiele für derartige Darstellungen finden sich in den während der 1850er Jahre erschienenen Naturgeschichten von Eduard Pöppig (1798-1868) und Christian Gottfried Giebel (1820-1881), deren Säugetierbände mit jeweils um die tausend Abbildungen dennoch als attraktives visuelles Angebot konzipiert waren (Pöppig 1851; Giebel 1859; Schwarz 2002: 185 f.). Insbesondere Giebel sprach bewusst einen weiteren Leserkreis an und bezog seine Illustrationen explizit auf das lebende Tier (Giebel 1859: 3). Tatsächlich gibt es bei Giebel einige Abbildungen, die durch ihre Lebendigkeit und Natürlichkeit überzeugen; in vielen von Giebels - und Pöppigs - Illustrationen wirken aber nicht nur die Tiere steif und unnatürlich, auch ihre Umwelt erscheint kulissenhaft, sofern sie überhaupt dargestellt ist (Finsch 1868: 65). Besonders auffallend sind die starken Qualitätsschwankungen, denen die Illustrationen in beiden Werken unterliegen und die auch deren grafische Umsetzung und den Druck umfassen. Weder Giebel noch Pöppig nannten die Namen von Illustratoren; auch die Abbildungen tragen weder die Signaturen ihrer Zeichner noch Beglaubigungen für ihre Naturtreue und stammen vermutlich aus recht unterschiedlichen Quellen (Gall 2011: $105 \mathrm{f}$.).

$\mathrm{Zu}$ den Kritikern solcher Zeichnungen gehörte der bereits erwähnte David Friedrich Weinland, der bei seiner Werbung für die neu errichteten zoologischen Gärten deren Vorzüge für den Künstler hervorhob: Dieser könne hier nämlich „mit Zuversicht schöpfen, ohne fürchten zu müssen, von verdrehten Formen mißleitet zu werden, wie es ihm auf dem Museum so oft geschieht, wovon die vielen schlechten Thierabbildungen sattsam Kunde geben“ (Weinland 1860b: 186, 1862; Hochadel 2011: 197). In anderem Zusammenhang plädierte er dafür, „den ganzen Formenwechsel, der an dem lebendigen Thierkörper zu Tage tritt, zu erfassen“. Solche Studien hätten nicht nur für die Erkenntnis des Seelenlebens der Tiere Bedeutung, sondern würden dem Zoologen auch bei der Unterscheidung nahe verwandter Arten oder der beiden Geschlechter helfen (Weinland 1863: 30). Tatsächlich ermöglichten es die zoologischen Gärten den Zeichnern, die Tiere über Stunden zu beobachten, ihre Bewegungsabläufe zu skizzieren und charakteristische Stellungen festzuhalten, während Präparate zwangsläufig auf eine einzige Haltung beschränkt waren (Voss 2005: 228).

Brehm teilte Weinlands Kritik und berichtete beispielsweise in seiner Gartenlauben-Reportage über das nordische Vogelleben, dass er beim Studium lebender Alken sehen konnte, „wie steif und kalt unsere besten Abbildungen sind“ (Brehm 1861a: 15). Es ist für die „biologische Perspektive“ bezeichnend, dass Brehm hier nicht etwa eine unzutreffende, untypische oder subjektive Darstellung kritisierte, sondern fehlende Lebendigkeit. Anders als Pöppig oder Giebel arbeitete Brehm über mehrere Jahre hinweg 
eng mit seinen Zeichnern zusammen, damit diese seine „Ansichten zu den ihrigen" machten, wie es im Vorwort des Thierlebens hieß (Brehm 1865a: VIII). Robert Kretschmer (1818-1872), dem er später die Verantwortung für sämtliche Illustrationen des Thierlebens übertrug, kannte er spätestens seit 1860, denn im November dieses Jahres schlug er der Naturforschenden Gesellschaft in Leipzig vor, diesen als ordentliches Mitglied aufzunehmen (Schneider 1988b: 62, Fn. 68). Kretschmer hatte an der Berliner Kunstakademie studiert und seit 1849 als leitender Illustrator bei der Illustrirten Zeitung in Leipzig gearbeitet. In den 1850er Jahren wandte er sich zunehmend dem Studium der Tier- und Pflanzenwelt zu und hatte 1856 mit dem Bild eines Kamels großen Erfolg auf einer Pariser Ausstellung (Anonym 1856). Bereits im Leben der Vögel lobte Brehm die enge Zusammenarbeit mit Kretschmer, der auf seine „Vorschläge bereitwillig eingegangen“ sei und ihm „,ortreffliche und richtige Zeichnungen geliefert" habe (Brehm 1861b: VII). Nach dessen Fertigstellung setzten sie ihre Zusammenarbeit offenbar bruchlos fort und machten den Zoo - ganz nach Weinlands Vorstellungen und englischem Vorbild - zum wichtigsten Ort von Kretschmers Arbeit. Im Oktober 1861, kurz nach der Publikation seines Vogelbuchs, schrieb Brehm jedenfalls an den befreundeten Botaniker und Ornithologen Carl Bolle (1821-1909), dass er zusammen mit Kretschmer einen Monat lang „in den Zoologischen Gärten Hollands, Belgiens und der Rheinlande“ gewesen und dieser inzwischen „ein vollendeter Thierzeichner geworden“ sei (Schneider 1988a: 55). Kretschmer präsentierte diese Zeichnungen im November 1861 der Naturforschenden Gesellschaft in Leipzig und zeigte sie auch Weinland, der von ihnen angeblich sehr angetan war (Schneider 1988a: 55; Weinland 1862: 20).

Während der hastigen Vorbereitungen für die Jagdexpedition Herzog Ernst II. zu Beginn des Jahres 1862 nutzte Brehm dann die Gelegenheit, Kretschmer für die noch offene Position des Reisemalers zu vermitteln (Haemmerlein 1993: 422 f.). Welcher Gewinn dadurch zu erwarten war, erkannte Roßmäßler, als er zur Abreise der Expedition schrieb, dass Kretschmer nicht bloß „Bilder aus dem dortigen Thierleben“ zeichnen werde, sondern auch die „dazugehörigen Pflanzenformen“: „Die von dem geschickten und gewissenhaften Maler zu verhoffenden Bilder werden uns also die Thiere nicht wie gewöhnlich in phantastischen Pflanzencoulissen verführen [sic! - vorführen?], sondern inmitten der wahren und wirklichen Pflanzennatur" (Roßmäßler 1862: Sp. 99-100). Dies kann man durchaus als Kritik an der Mehrzahl der zeitgenössischen Tierabbildungen lesen, auf denen die Tiere selbst zwar mittlerweile "nach dem Leben“ gezeichnet sein mochten, ihre Umgebung aber meist „hinzuphantasiert“ war. Implizit brachte Roßmäßler damit den Anspruch zum Ausdruck, dass 
Naturtreue und Augenzeugenschaft im Idealfall die gesamte Darstellung umfassen sollten und nicht nur Teile davon.

In den beiden Säugetierbänden des Thierlebens finden sich allerdings nur vier Abbildungen Kretschmers (Brehm 1864: 80, 1865a: 611 f., 688, 722), die mit Sicherheit unter dem unmittelbaren Eindruck der ostafrikanischen Tierwelt entstanden sind. Denn sie illustrierten bereits eine Artikelserie Brehms über die Abessinien-Expedition (Brehm 1863b: 168, 297, 328, 329). ${ }^{4}$ Im Thierleben waren immerhin drei dieser Abbildungen als ganzseitige Bildtafeln gestaltet, die in jeden Band eigens eingebunden waren und eine besonders hohe Druckqualität aufwiesen (Gall 2011: 106-108). ${ }^{5}$

Von den 402 direkt in den Text eingefügten Abbildungen der beiden Säugetier-Bände tragen jedoch nur 146 Kretschmers Signatur, 112 stammen von dem Tiroler Künstler Theodor Franz Zimmermann (1808-1880), mit dem Brehm auch anderweitig zusammenarbeitete (Brehm \& Roßmäßler 1864) und dessen Begabung er ebenfalls schätzte (Brehm 1862: 589, 1865b: V f.). ${ }^{6}$ Zudem war Zimmermann, zumindest anfangs, auf den Titelblättern der einzeln über den Kolportagevertrieb ausgelieferten Hefte des Illustrirten Thierlebens neben Kretschmer namentlich als Illustrator genannt (Haemmerlein 2015: 64). ${ }^{7}$ Warum sein Name auf den Titelseiten der Buchhandelsausgabe dann keine Erwähnung mehr fand, ist unklar.

Wie Brehm im Vorwort betonte, hatten Kretschmer und Zimmermann angegeben, „ob sie das lebende Thier gezeichnet, oder nur eine gute Abbildung benutzt" hatten (Brehm 1865a: VIII). Beide hatten ihre Zeichnungen in der Regel mit ihrer Signatur versehen, diese im ersten Fall aber noch um den Zusatz „n[ach] d[em] Leben“ oder „n[ach] d[er] Natur“ ergänzt, um die Authentizität der eigenen Beobachtung nachzuweisen. Damit entsprachen diese Illustrationen Brehms Konzept, das lebende Tier in den Mittelpunkt des Thierlebens zu stellen. Der Verlag machte daraus umgehend ein Verkaufsargument und warb schon in der ersten Ankündigung des Werks damit, „daß Alles und Jedes nach dem Leben gezeichnet wurde“ (Sarkowski 1976: 88). Die ersten Rezensionen lobten denn auch „die beigegebenen Bilder in Holzschnitten“: „Viele davon“ seien „Originale nach dem Leben, was leider von so wenigen der publicirten Thierbilder gesagt werden“ könne (Anonym 1863a: 114). Brehm übte sich dagegen in Selbstkritik und beklagte, dass das Thierleben mehr "fehlerhafte Abbildungen“ enthalte, „als wir im Voraus fürchten konnten“. Die Schuld daran gab er der Unmöglichkeit, die betreffenden Tiere lebend zu beobachten, wodurch er indirekt noch einmal seine Ansprüche an gelungene Illustrationen bekräftigte (Brehm 1865a: VIII). ${ }^{8}$

Neben den von Kretschmer und Zimmermann signierten Abbildungen enthält der erste Säugetierband noch 85 und der zweite weitere 56 ohne Signatur. Das war insofern nicht erstaunlich, als Brehm angeblich schon 
im Voraus damit gerechnet hatte, dass er und seine Künstler „genöthigt sein würden, nachzuschreiben und nachzubilden“ (Brehm 1865a: VIII). Aber anders als im Vorwort suggeriert und anders als es Brehm bei direkten oder indirekten Zitaten in der Regel selbst handhabte (Haemmerlein 1989: 24-27; Schulze 2009: 154-219), sind bei den unsignierten Abbildungen überwiegend keine Quellen angegeben. ${ }^{9}$ Tatsächlich stammen sie zum allergrößten Teil aus der Illustrated Natural History des englischen Wissenschaftspopularisierers John George Wood (1827-1889), der ein inhaltlich ähnliches Konzept wie Brehm verfolgte. ${ }^{10}$

Obwohl die unsignierten Illustrationen im Thierleben bei der Übernahme aus Woods Werk meist etwas von ihrer Qualität einbüßten, ergibt sich ein bemerkenswert einheitlicher Gesamteindruck. Das mag zum einen daran liegen, dass auch Kretschmer und Zimmermann nur teilweise nach lebenden Tieren zeichneten und häufig auf ganz unterschiedliche Vorlagen zurückgreifen mussten, die unter Umständen ungenügend waren. ${ }^{11}$ Zum anderen, und das ist im vorliegenden Zusammenhang zentral, geben nahezu alle Illustrationen in mehr oder weniger detaillier Ausführung die natürliche Umgebung der dargestellten Tiere wieder, und zwar unabhängig davon, unter welchen Umständen und mit welchen Vorlagen die Zeichnungen entstanden sind. Zu den wenigen Beispielen, an denen dies explizit deutlich wird, gehört die Darstellung des Onagers, einer Unterart des asiatischen Wildesels, bei der Brehm angibt, dass Zimmermann sie nach dem Vorbild eines Eselspaares im Tiergarten Schönbrunn angefertigt habe (Brehm 1865a: 362, 364). Die Abbildung (Abb. 1) trägt unter Zimmermanns Signatur den Zusatz „n. d. Leben“, zeigt aber statt eines Eselpaares vier Tiere und statt des Zoogeheges eine Berglandschaft. Selbst wenn sich Zimmermann eigens über das natürliche Verbreitungsgebiet des Onagers informiert haben sollte, entstammt der Hintergrund seiner künstlerischen Imagination und fällt damit in die Kategorie der von Roßmäßler kritisierten „Pflanzencoulissen“.

Die Darstellung der natürlichen Umgebung der Tiere auf den Abbildungen des Thierlebens hatte nicht nur eine deutliche Differenz zu den Abbildungen der universitären Zoologie zur Folge, die den Lebensraum der Tiere allenfalls andeutete und seit den 1850er Jahren meist ganz davon absah (Blum 1993: 48, 195), sondern zwangsläufig auch die Ausblendung des Zoos und damit des Ortes, an dem die Zeichner die Tiere meist studiert hatten: Statt der Gehege zeigten die Illustrationen Thierlebens also eine imaginierte Natur, um auf diese Weise - so meine These - den von der „biologischen Perspektive“ geprägten Vorstellungen Brehms gerecht zu werden. Konventionen oder persönliche Vorlieben der Zeichner waren jedenfalls nicht die Ursache, denn in anderen Arbeitszusammenhängen schuf Zimmermann durchaus Zeichnungen, die Tiere im Umfeld des 


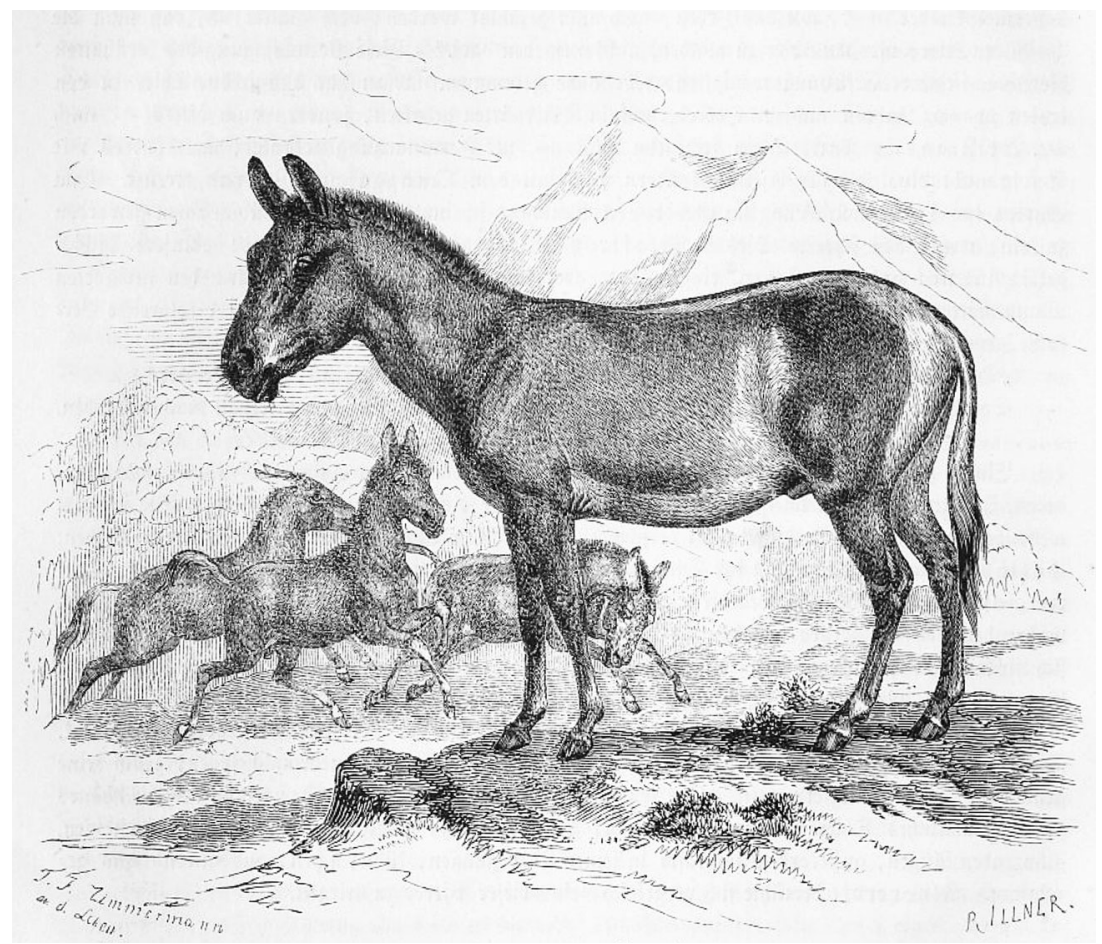

Abb. 1 Als Vorbild für seine Zeichnung „Onager" diente Theodor F. Zimmermann das Eselspaar im Tiergarten Schönbrunn, er versetzte die Tiere aber in eine Gebirgslandschaft, die deren Lebensraum in Südwestasien wiedergeben soll. Holzstich von Richard Illner (Brehm 1865a: 362)

Zoos zeigten (Brehm \& Zimmermann 1865). Außerdem war der Zoo mit seinen Tieren vor allem in den Illustrationen der Wochenzeitungen regelmäßig präsent und den Zeichnern gelang es dabei durchaus, auch deren gattungs- und artspezifischen Merkmale anschaulich wiederzugeben (Gall 2011: $110 \mathrm{f}$.).

Für eine breite Leserschaft war es jedoch reizvoll, Tiere auf den Illustrationen nicht isoliert oder in Zoogehegen, sondern in ihrem Lebensraum zu betrachten. So erinnerte sich etwa der Zoologe Ludwig Heck (1860-1951), wie er als Elffähriger beim Durchblättern des Thierlebens die „Tiere in ihrer natürlichen Umgebung, ihrer heimischen Landschaft dargestellt und dadurch zu vollem Leben erweckt" sah (Heck 1938: 343; Blum 1993: 48). Diese Form der Darstellung hatte aber auch ihren Preis, denn die Zeichner gingen damit das Risiko ein, ihrer Phantasie allzu freien Lauf zu lassen und die Tiere in einer Umwelt zu zeigen, die in deren Verbreitungsgebiet gar nicht existierte. So wies der berühmte Berliner Arzt, Wissenschaftler und Politiker Rudolf Virchow (1821-1902) noch in seiner Rezension der 


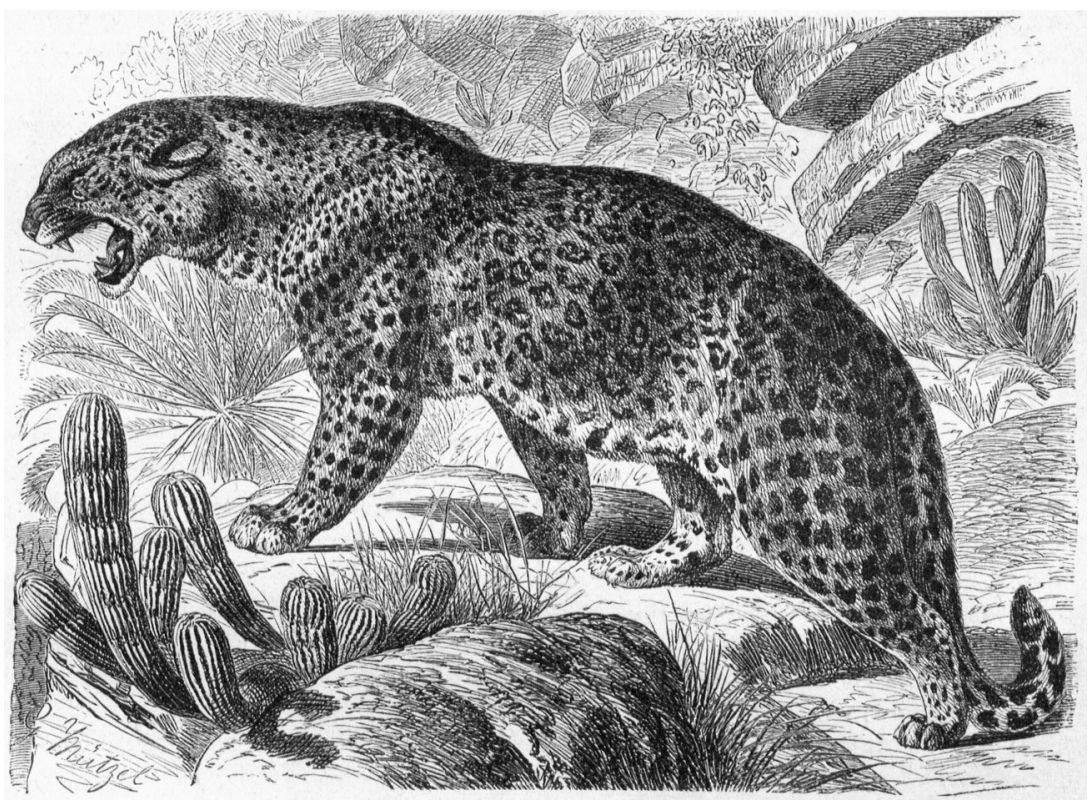

Abb. 2 Gustav Mützels Zeichnung eines Leoparden entstand "n. d. Leben“ in einem Zoo, zeigt inn aber in einer Halbwüste im Umfeld von Kakteen. Rudolf Virchow kritisierte, dass eine derartige Flora in der Heimat des Leoparden nicht vorkäme. Holzstich von Richard Illner (Brehm 1876: 423; Pechuël-Loesche 1890: 462; Virchow 1890: 171)

dritten, überarbeiteten und verbesserten Auflage des Thierlebens von 1890 auf einen „gewissen Error loci“ hin, „der sich bei manchen Abbildungen störend bemerkbar" mache und kritisierte „die Ausschmückung der Thierbilder mit Pflanzen ganz anderer Gegenden, ja anderer Continente, als der Heimath der dargestellten Thiere. Beispielsweise sei auf die Bilder des Leoparden (S. 462) [Abb. 2], des Karakal (S. 515, Tafel) und des Ichneumon (S. 567, Tafel) hingewiesen. Schwerlich dürfte sich in der Nähe der Pyramiden eine Vegetation vorfinden, wie sie hier dem Ichneumon zugedacht ist" (Virchow 1890: 171).

Die von Virchow kritisierte Zeichnung des Leoparden stammt von Gustav Mützel (1839-1893) und war erstmals in der zweiten, erweiterten Auflage des Thierlebens erschienen (Brehm 1876: 423). Bei der Neubearbeitung wurde sie von Eduard Pechuël-Loesche (1840-1913) auch in die dritte Auflage übernommen (Pechuël-Loesche 1890: 462). Anders als Kretschmer hatte Mützel nie an einer Expedition teilgenommen und kannte exotische Tiere nur aus dem Zoo (Müller-Liebenwalde 1893: 326). Nach Hauffe und Klös (1995: 32-38) setzte er die Umgebung auf seinen Tierzeichnungen deshalb häufig aus Versatzstücken zusammen, für die er unterschiedliche Vorlagen wie Fotos, Zeichnungen, Aquarelle oder Expeditionsobjekte nutz- 
te. Zusätzliche Kenntnisse fremdländischer Flora und Geographie eignete er sich darüber hinaus bei der Illustration zahlreicher populärwissenschaftlicher Reisewerke an.

Im Unterschied zu Virchow waren die meisten Rezensenten bei der Besprechung der ersten Auflage weniger kritisch und betonten wie etwa Brehms Freund Carl Bolle (1863), dass „die beigegebenen zahlreichen Abbildungen und in den Text eingedruckten Holzschnitte [...] grossentheils nach dem Leben in der Wildniss [sic!] entnommenen Skizzen ausgeführt" seien. Otto Ule (1820-1876) meinte ihnen immerhin anzusehen, „daß sie nach dem Leben auf Reisen oder in zoologischen Gärten gezeichnet wurden“ (Anonym 1863b: 12). Nur dem Genfer Geologen und Zoologen Karl Vogt (1817-1895) war es nicht entgangen, dass in manchen Zeichnungen einzelne Partien der Phantasie der Künstler entsprungen waren. Er lobte deshalb, dass ihre "landschaftliche Staffage mit Treue und Sorgsamkeit ausgewählt und harmonisch der Thiergruppe angefügt" worden sei. ${ }^{12}$ So selbstkritisch Brehm sich im Vorwort gegenüber den Abbildungen seines Werkes gegeben hatte, an ihrer landschaftlichen "Staffage“ nahm er offenbar keinen Anstoß.

\section{Dramatik und Attraktivität zoologischer Illustrationen in der Gartenlaube}

Brehm gehörte, wie oben erwähnt, seit 1858 zum Autorenkreis der Gartenlaube. Sie wies in den Jahrzehnten nach ihrer Gründung 1853 die höchste Auflage unter allen deutschen Zeitschriften und Zeitungen auf und blieb bis zum Ende des Jahrhunderts das Leitmedium in Deutschland (Graf \& Pellatz 2003; Faulstich 2004: 65-74). Mit ihrer Gründung hatte sich Ernst Keil auch das Ziel gesetzt, die Naturwissenschaften zu popularisieren. Allein Brehm lieferte ihm in knapp 20 Jahren mehr als 70 Beiträge (Daum 2002: 338 f.; Haemmerlein 2015: 56). Obwohl ihr Untertitel sie als „Illustrirtes Familienblatt“ auswies, lag der Gesamtanteil der Abbildungen zunächst bei nur etwa 15 Prozent. Erst nach ihrem Verkauf an den Kröner Verlag im Jahr 1883 erhöhte er sich deutlich und stieg auf über 40 Prozent (Graf \& Pellatz 2003: 472).

Welche Bedeutung Illustrationen bei der Popularisierung der Zoologie in der Gartenlaube besaßen, zeigt der Vergleich mit anderen Themen aus dem Spektrum Naturkunde, Medizin, Geographie und Technik. So lag die Zoologie gemessen an der Anzahl der im Zeitraum von 1890 bis 1905 publizierten Artikel hinter der Medizin an zweiter Stelle mit deutlichem Abstand vor der Botanik. Während aber von den botanischen Beiträgen 
nur gut die Hälfte (95 von 174) und von den medizinischen Beiträgen sogar nur ein gutes Viertel (135 von 488) illustriert waren, wiesen bei der Zoologie mehr als drei Viertel (329 von 427) mindestens eine Abbildung auf. Zusätzliches Gewicht erhält dieses Ergebnis durch die bei der Zoologie häufige Trennung von Bildern und Texten in der Weise, dass einer großen doppel-, ganz- oder halbseitigen Abbildung im vorderen Heftteil meist ein kürzerer Text am Ende der Ausgabe folgte. Während bei den anderen Themen aus Naturkunde, Medizin, Geographie und Technik eine derartige Trennung nur in Ausnahmefällen vorkam, war sie bei Kunstreproduktionen und Genrebildern seit den 1870er Jahren üblich (Schöberl 1996: 228-232). ${ }^{13}$ Das lässt durchaus den Schluss zu, dass die Redaktion der Gartenlaube zoologische Illustrationen für ähnlich attraktiv hielt wie die Abbildung von Kunst. ${ }^{14}$

Viele der Beiträge Brehms in der Gartenlaube erschienen anfangs zusammen mit Abbildungen, die von Kretschmer oder Zimmermann stammten. Auf diese Weise übernahm die Zeitschrift den illustrativen Stil des Thierlebens und stellte die abgebildeten Tiere überwiegend in ihrer natürlichen Umgebung dar. Darüber hinaus gab es aber auch einige weitere Darstellungsweisen, unter denen dramatische Kämpfe zwischen Tieren besonders auffallen. Diese Auffälligkeit ist allerdings weniger auf ihre Gesamtanzahl zurückzuführen, da der Anteil derartiger agonaler Tierbilder in der Gartenlaube zwischen 1880 und 1900 bis auf zwei Ausnahmejahre stets unter einem Viertel lag. Wichtiger war ihr meist doppel- oder ganzseitiges Format und ihre durchgängige Platzierung auf der rechten Zeitschriftenseite (bei ganz- oder halbseitigem Format), so dass sie auch bei oberflächlicher Betrachtung kaum zu übersehen waren. Außerdem besaßen die übrigen Illustrationen der Gartenlaube einen eher heiteren, erbaulichen und harmonischen Charakter, der in deutlichem Kontrast zur Darstellungen kämpfender Tiere mit ihrer Dramatik und Spannung stand (Wildmeister 1998: 129-132).

Ein eindrückliches Beispiel für die gezielte Dramatisierung einer Tierkampfdarstellung bietet eine Zeichnung Friedrich Spechts (1839-1909), die die Gartenlaube 1883 unter dem Titel „Des Tigers Beute“ druckte (Abb. 3). In ihrer xylographischen Reproduktion zeigt sie einen indischen Büffel, der - vor einer Dschungelkulisse - im Wasser stehend von einem Tiger angefallen wird und kaum noch Chancen hat, dem Angriff zu entkommen. Der Fokus liegt auf dem aufgerissenen Maul des Büffels, seinen angstgeweiteten Augen und den aufblitzenden Zähnen des Tigers. Verstärkt wird die Dramatik des Kampfes durch die kontrastreiche Beleuchtung des Zentrums, die beiden entfliehenden Vögel, die von den Körpern der Kämpfenden wegspritzenden Wassertropfen und die diagonale Anordnung von Beute und Räuber, die dem Bild zusätzliche Dynamik verleiht. Die Zeich- 
Abb. 3 Friedrich Specht stellte den Kampf eines Tigers mit einem indischen Büffel vor einer Dschungelkulisse dar und nutzte unter anderem die Lichtführung, um die Dramatik der Szene zu verstärken. Holzstich von Carl Gottlob Specht (Anonym 1883)

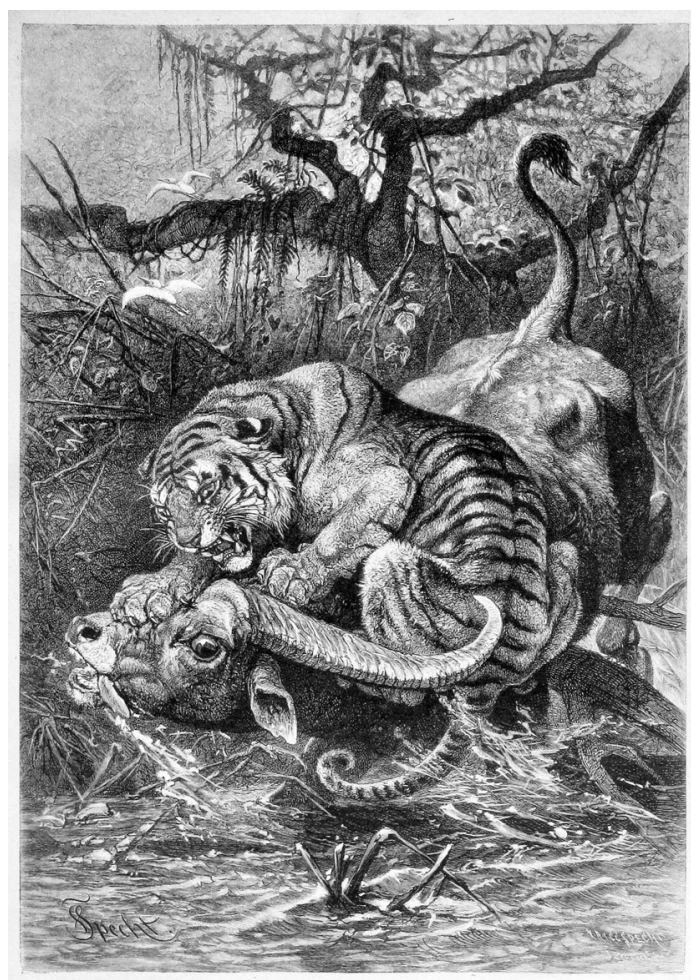

nung gibt sich damit leicht als Inszenierung zu erkennen; zudem können wir mit großer Wahrscheinlichkeit davon ausgehen, dass Specht weder den indischen Dschungel bereist, noch eine derartige Szene selbst beobachtet hatte. ${ }^{15}$ Schlawe (1994: 44) unterstellt ihm sogar, „eigentlich nur von der Orientierung an fremden Vorbildern" gelebt zu haben, während ein zeitgenössischer Rezensent die außerordentliche Qualität von Spechts Zeichnungen immerhin auf seine "Studien an dem lebenden Tiere“ in den Zoologischen Gärten zurückführte (Noll 1884: 29). Die Gartenlaube attestierte Spechts Tigerzeichnung jedenfalls eine Darstellung „in seltener Lebenstreue“ (Anonym 1883: 692).

Anders als die Arbeiten von Kelly (1981: 22-34), Schwarz (1999: 276) und Voss (2009: 252-254) erwarten lassen, diente die Publikation agonaler Tierbilder in der Gartenlaube nur selten einer Vermittlung von Darwins Theorien oder verwandten Vorstellungen. ${ }^{16}$ Ihre Aufgabe bestand vielmehr darin, die Aufmerksamkeit der Leser zu wecken. Im Vergleich zum Buchmarkt standen die Zeitschriften mit ihren regelmäßigen Erscheinungsterminen nämlich unter einem höheren Druck. Als in den 1890er Jahren die Auflage der Gartenlaube zu sinken begann und die Konkurrenz wuchs, wurde es für sie deshalb immer wichtiger, Leser und Abonnenten mit 
attraktiven und großformatigen Bildern in hoher Qualität für sich zu gewinnen, ihre Bildungs- und Unterhaltungsbedürfnisse zu befriedigen und dadurch eine möglichst regelmäßige Nachfrage zu stimulieren (Beetham 1990: 21).

\section{Landschaftskulissen im Tierpark: Ottomar Anschütz' Momentfotografien}

Vergleicht man die xylographische Bilderwelt der Familienzeitschriften und illustrierten Zeitungen vor der Jahrhundertwende mit der fotografischen nach der Jahrhundertwende, dann scheint dem ersten Eindruck nach der Unterschied auf keinem anderen Gebiet so groß gewesen zu sein wie bei der Zoologie. Von Personen, Architektur, Technik, Pflanzen und vielen wissenschaftlichen Phänomenen wurden seit Mitte der 1870er Jahren nämlich zunehmend Photoxylographien angefertigt, die Fotografien durch eine Kombination fotochemischer und manueller Techniken als Holzstich reproduzierten und damit deren Ästhetik zumindest ansatzweise in die Familienzeitschriften und illustrierten Zeitungen transportierten (Weise 2003: 80-93). Reproduktionen von Tierfotografien blieben jedoch eine Ausnahme, da es in diesem Zeitraum ohnehin nur vergleichsweise wenige Aufnahmen von lebenden Tieren gab und diese sich auf statische Positionen und Profilansichten beschränkten (Pohlmann 2004b). Der Hauptgrund dafür lag in der Unempfindlichkeit der fotografischen Platten und der unausgereiften Mechanik der Kameraverschlüsse, die kurze Belichtungszeiten, wie sie für eine lebendige Darstellung notwendig gewesen wären, kaum zuließen.

Anstelle lebender Tiere war deshalb die Aufnahme von präparierten Tieren in freier Natur seit Ende der 1850er Jahre eine immer wieder anzutreffende Praxis. Allerdings ist in der Forschung umstritten, ob die Tierpräparate auf derartigen Fotografien tatsächlich lebende Tiere in ihrer Umwelt simulieren sollten (Ryan 1997: 114-117; Lechtreck 2005: 78-80) oder ob sie nicht eher als pittoreske Kompositionselemente romantischer Landschaftsdarstellungen $\mathrm{zu}$ verstehen sind (Knoepfelmacher \& Tennyson 1977: xxi; Millard 1977: 25; Brower 2010:1-24). Mit der Entwicklung schnellerer Platten und präziserer Verschlüsse büßte diese Praxis an Akzeptanz ein und galt spätestens nach der Jahrhundertwende als „Unfug“ oder vorsätzliche Täuschung (Lechtreck 2005: 77-84; Meerwarth 1905: 3).

Vor der Wende zum 20. Jahrhundert beherrschten jedoch nur wenige Spezialisten die Technik der sogenannten Momentfotografie soweit, dass scharfe und beeindruckende Aufnahmen von lebenden Tieren entstanden. 
In Deutschland gehörte dazu der aus Posen stammende Ottomar Anschütz. Eine Voraussetzung für seine Momentaufnahmen waren lichtempfindlichere Trockenplatten, die das aufwendige Kollodiumverfahren ablösten, eine andere schuf Anschütz selbst, als er in den 1880er Jahren einen präzise funktionierenden Schlitzverschluss für kurze Belichtungszeiten bis zu 1/1000 Sekunde konstruierte (Rossell 2001: 16-20). Neben Militärmanövern zählten lebende Tiere dabei zu seinen bevorzugten Motiven; insbesondere seine Aufnahmeserie einer Storchenfamilie aus dem Jahr 1884 verschaffte ihm allgemeine Bekanntheit. Anschütz hatte sich dafür in der Nähe ihres Nests postiert und fotografierte die Vögel unter anderem bei Anflug, Landung oder der Fütterung ihrer Jungen (Müllenhoff 1885/1886: 396-399; Rossell 2001: 27 f.).

In den folgenden Jahren griff Anschütz bei seinen Tierfotografien meist stärker in den Aufnahmeprozess ein, um größeren Einfluss auf das Ergebnis zu nehmen. So band er ein Zicklein an einem Pflock fest und ließ dann einen Wolf los, um die tödlich endende Begegnung in einer Bildserie festzuhalten (Vogt 1886: Sp. 335-338). Ihre „absolute Naturtreue“ und ihre „ästhetischen Vorzüge" wurden ausführlich in einem feuilletonistischen Artikel über die „Augenblicksphotographie“ behandelt. Ja, der Autor verglich ihre Qualitäten sogar mit denen des „Künstlerauges“ und erklärte sie zur Richterin über die Wahrheit der Tiermalerei (Müller 1887: 68 f.).

Während man den Wolfsbildern ihre Inszenierung leicht ansah, trieb Anschütz Ende der 1880er Jahre bei einer Serie von Raubkatzenaufnahmen einen enormen Aufwand, um auf den fertigen Fotografien die äußeren Eingriffe $\mathrm{zu}$ verbergen. Im Breslauer Zoo hatte er dazu einen nach oben offenen, 140 qm großen Zwinger mit steppenartigem Untergrund errichten lassen, während als Hintergrund eine gemalte - auf dem Foto aber weitgehend unscharf erscheinende - Landschaft diente. Gemalte Hintergründe waren in der Portraitfotografie des 19. Jahrhunderts zwar schon lange üblich (Herrmann 2014: $33 \mathrm{f}$.), Anschütz nutzte sie aber für ein neues Genre und in deutlich größeren Dimensionen. ${ }^{17}$ Für die Momentaufnahme von zwei Geparden (Abb. 4) benötige er außerdem das volle Sonnenlicht, weshalb er die Tiere erst hungern ließ, um sie dann mit Futter gezielt an die hellste Stelle zu locken. Wie bei seinen Wolfsbildern verwendete Anschütz dafür „lebende Nahrung“, um in seinen Aufnahmen „grösste Naturwahrheit und einen erhöhten Affect zu erzielen“ (Anschütz 1889).

Anschütz griff damit auf die gleichen visuellen Strategien zurück, die vor ihm schon die Tierzeichner genutzt hatten: Er blendete den Zoo aus und versetzte die Tiere scheinbar in ihr natürliches Umfeld, das allerdings zur Gänze künstlich gestaltet war. Außerdem manipulierte er das Verhalten der Raubkatzen, um seinen Aufnahmen - ähnlich den Illustrationen der Zeitschriften - zusätzliche Dramatik zu verleihen. Mit den populären zoo- 


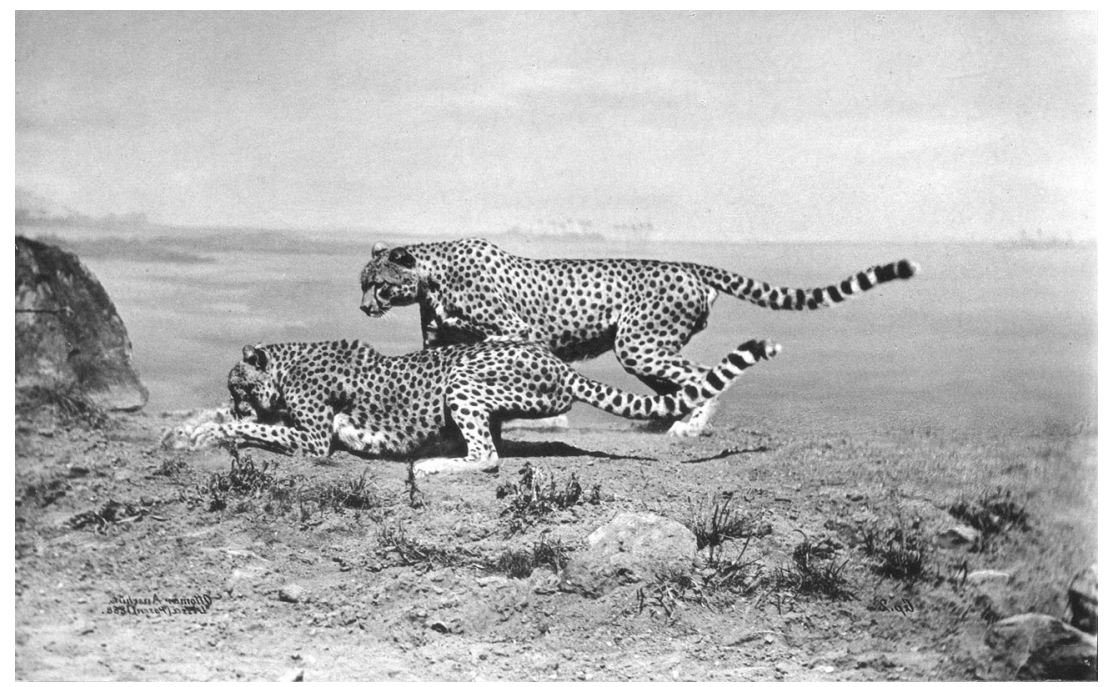

Abb. 4 Ottomar Anschütz nahm die "Gruppe von Gepards" 1888 im Zoologischen Garten von Breslau in einem speziellen Zwinger vor einem gemalten Hintergrund auf. Seitenverkehrte Vergrößerung bereits in der Publikation (Anschütz 1889)

logischen Illustrationen war Anschütz schon aufgrund ihrer großen Verbreitung vermutlich gut vertraut. Darüber hinaus stellte er den direkten Bezug zu ihnen her, als er, ebenso wie viele seiner Kritiker, seine Aufnahmen „als Studienmaterial für Künstler und alle Kunstlehranstalten“ empfahl (Anschütz 1889; Müller 1887: 68 f.; Noll 1889: 136). ${ }^{18}$ Man kann also davon ausgehen, dass Anschütz sich an der populären Tierzeichnung orientierte und mit der Integration von natürlicher Umgebung und expressiver Lebendigkeit auch wesentliche Merkmale der "biologischen Perspektive“ in seine Fotografien übernahm. Trotz aller Inszenierung sollten sie aber zugleich als Illustrationen für naturwissenschaftliche Lehrbücher dienen, „da ein so wahres Bild [...] keine Menschenhand wiederzugeben im Stande“ sei (Anschütz 1889).

Mit seinen Aufnahmen folgte Anschütz keiner tierkundlichen Systematik, er stellte sie nur für Ausstellungen zusammen und verkaufte sie im Selbstverlag einzeln oder in Mappen. Außerdem wurden seine fotografischen Serien sowie etliche Einzelaufnahmen in zahlreichen nationalen und internationalen Zeitschriften reproduziert und in Einzelfällen tatsächlich, wie von ihm vorgesehen, zur Illustration naturkundlich orientierter Bücher verwendet. $\mathrm{Zu}$ diesen zählte die 1897 erschienene Naturgeschichte und Charakterschilderung der zur hohen Jagd gehörigen Thiere Mitteleuropas des Arztes Wilhelm Wurm (1831-1913), bei der es sich wohl um das erste deutsche tierkundliche Buch mit durchgängig fotografischen Illustrationen handelt. In mehrfacher Hinsicht folgte es der „biologischen 
Perspektive“: So war es durchaus typisch, dass Wurm als Autor nicht der universitären Zoologie angehörte, aber gelegentlich im Zoologischen Garten publizierte. ${ }^{19}$ Außerdem wollte er über den Jäger und den gelehrten Forscher hinaus, auch den bildenden Künstler, den Museumsbeamten und Präparator sowie das große gebildete Publikum ansprechen. Sodann legte er besonderen Wert „auf die Hervorhebung der biologischen und namentlich der psychischen Seite des Thieres“, die seiner Meinung nach bisher sehr vernachlässigt worden war. Und schließlich führte Wurm dieses Defizit, ähnlich wie etwa Brehm, auf den fehlenden Umgang der meisten Forscher mit lebenden Tieren zurück (Wurm 1897: 1, Hervorh. i. Orig.).

Davon abgesehen ist das Buch ein Beispiel für die Schwierigkeiten, vor der Jahrhundertwende eine größere, nach zoologischen, regionalen oder anderen Kriterien ausgewählte Tiergruppe wenigstens annähernd systematisch mit Fotografien zu illustrieren und nicht bloß gelungene Einzelaufnahmen als Trophäen eines fotografischen Abenteuers zu präsentieren. Anschütz' Fotografien deckten nämlich nur einen kleinen Teil der beschriebenen Jagdtiere $a b$, weshalb sich der Verlag an die Leser fotografischer Fachzeitschriften gewandt hatte, um Lücken zu schließen und die Auswahl an Bildern zu vergrößern (Anonym 1897). Dennoch enthielt Wurms $\mathrm{Na-}$ turgeschichte viele Aufnahmen von unzureichender Qualität, mit starken Retuschen sowie möglicherweise auch von präparierten Vögeln in freier Natur (Guggisberg 1977: 43 f.). Dementsprechend scheiterte ein ursprünglich geplanter zweiter Band über die Tiere der niederen Jagd bereits daran, die dafür notwendigen Fotografien zu beschaffen (Wurm 1897: 232).

\section{Zootiere in retuschierter Natur: Ludwig Hecks Lebende Bilder}

Während die Aufnahme präparierter Tiere in freier Natur in den 1890er Jahren also immer weniger als ernstzunehmende fotografische Praxis in Frage kam, hatten Anschütz' Momentbilder zwar großen Erfolg und galten in mehrfacher Hinsicht als Vorbild, einen vergleichbaren Aufwand wollten oder konnten jedoch nur die wenigsten Fotografen treiben. Alternativ bot sich die direkte Aufnahme von Tieren im zoologischen Garten an, ohne die Konstruktion eigener Fotogehege. Dass derartige Bilder zumindest in Großbritannien für ein breites Publikum attraktiv sein konnten, zeigte etwa das 1895 erschienene und mehrfach wiederaufgelegte Werk Life at the Zoo des Naturkundeautors Charles John Cornish (1896). In Deutschland diente es Ludwig Heck als Vorbild, um ein vergleichbares Werk für den Berliner Zoo zu schaffen, dessen Direktor er war (Schütt 1895). Nach mehrjähriger Vorbereitungsphase erschien es 1899 unter dem Titel Lebende Bilder aus 
dem Reiche der Tiere im großen „Prachtwerk-Format“ mit „200 MomentAufnahmen nach dem Leben“. ${ }^{20}$ Diese Aufnahmen waren vermutlich in langwieriger Zusammenarbeit mit einem einzelnen oder einigen wenigen ungenannt bleibenden Fotografen entstanden.

Mit dem „Prachtwerk“ wollte Heck den Tierbestand seines zoologischen Gartens „zur naturgeschichtlichen Anschauung und Volksbildung“ nutzen. Diese Schlagwörter waren um die Jahrhundertwende weit verbreitet und gehörten unter anderem zum typischen Vokabular der Museumsreformer. Sie warben damit beispielsweise für die Aufstellung biologischer Gruppen und Dioramen, um einem breiten Publikum die Lebensweise der dargestellten Tiere näher zu bringen (Nyhart 2009: 251-292; Köstering 2003: 226-245). Und so wie die Museumsreformer die langen taxonomischen Reihen aus Tierpräparaten ablehnten, so lehnte Heck die „trockenen, langatmigen Kopf-Schwanz-Beschreibungen“ ab, die seiner Meinung nach nur für Fachleute notwendig waren, für den Tierfreund aber „so gut wie nutzlos“. Stattdessen forderte er "Schilderungen des lebenden Tieres“. Dabei bezog er sich auf Brehm, mit dem er nicht nur die Stellung als Zoodirektor und den Glauben an eine Tierseele teilte (Daum 2002: 435), sondern auch die „biologische Perspektive“. Dass dessen Thierleben „den Nachdruck auf das ,Leben“" gelegt hatte, hatte Heck zufolge „einen neuen Abschnitt in der Geschichte des gemeinverständlichen zoologischen Schrifttums" eingeleitet. Ähnlich wie die Museumsreformer spielte er mit dem Begriff „Leben“ das Bild gegen das Wort aus, welches „Leben“ „immer nur in beschränktem Maasse [sic!] bieten“ könne, und dann die Fotografie gegen die Zeichnung: Denn selbst den besten Illustratoren unterliefen immer wieder „Verzerrungen“, während der Fotograf „das Tier in lebensvoller und ihm eigentümlicher Stellung auf seiner lichtempfindlichen Platte festbannt“. Mit den „Tierabbildungen von dieser Grösse, unmittelbar vom lebenden Urbild genommen“, wandte sich Heck an einen breiten - und damit den für die „biologische Perspektive“ typischen - Adressatenkreis und hoffte „dem Gelehrten, dem Künstler und Tierfreund, der Schule und der Familie“ etwas wirklich Neues bieten zu können (Heck 1899: 3 f.).

Allerdings enthielt das Buch, das 1909 in zweiter Auflage erschien, nur wenige Abbildungen, die die Tiere mit der vollen Schärfe und der Plastizität zeigten, die dem Charakter der Fotografie als wissenschaftlichem Illustrationsmedium entsprochen hätte. Aus den Bildunterschriften lässt sich schließen, dass die langen Belichtungszeiten bessere Ergebnisse vielfach verhindert hatten. Ein Großteil der Abbildungen weist deshalb mehr oder weniger starke Retuschen auf, die meist dazu dienten, die Schärfe zu erhöhen oder störende Details zu entfernen (Peters 2004: 242).

In einigen Fällen verfolgten die Retuschen jedoch eindeutig das Ziel, die Tiere ohne die Gehege des Zoos gleichsam in ihrer natürlichen Umgebung 


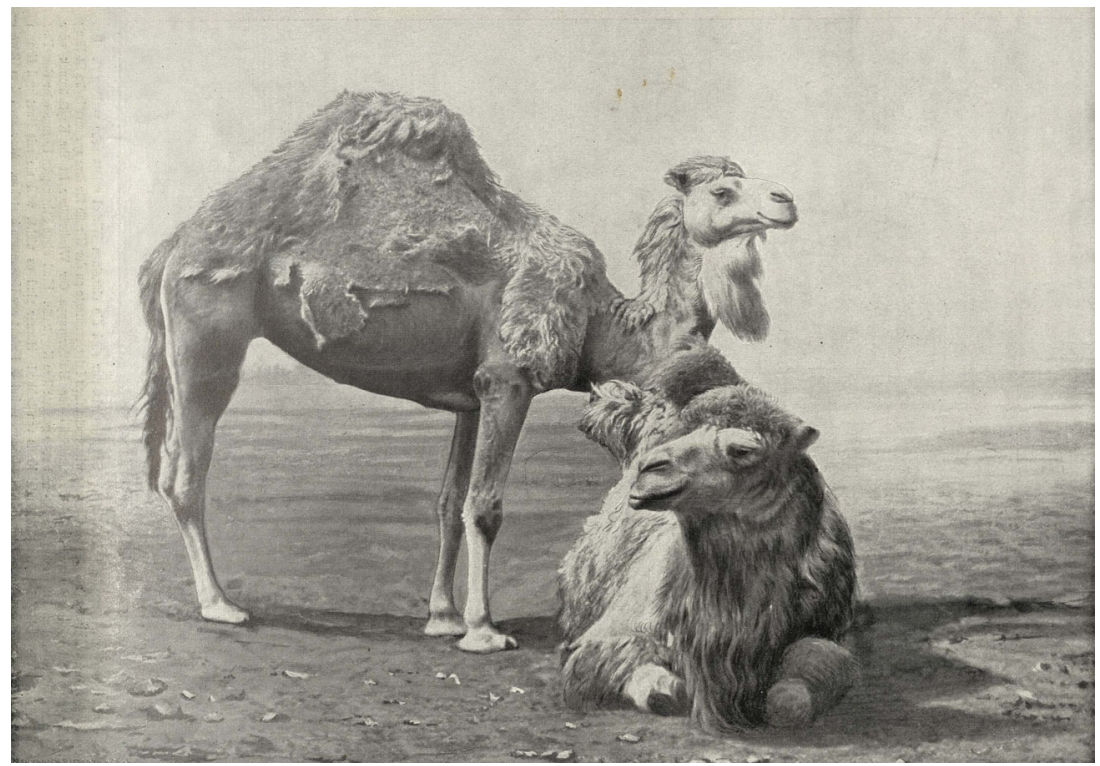

Abb. 5 Der Retuscheur übermalte bei dem Bild "Dromedar und Kamel“ jeden Hinweis auf den Zoo als den Ort der Aufnahme mit einer Wüstenlandschaft, die auf den natürlichen Lebensraum der Tiere verweist (Heck 1899: 7)

zu zeigen. So wies Heck bei der Abbildung eines Dromedars und eines Kamels (Abb. 5) auf seinen "genial angehauchte[n] Retoucheur" hin, der „die ganze Umgebung bis zum verschwimmenden Horizont" so hergerichtet habe, dass „die beiden mächtigen Tiere ganz stimmungsvoll als ,Schiffe der Wüste“ erscheinen“ müssten. Seinen mit dem neuen Medium noch unvertrauten Lesern versicherte er zugleich, dass auch diese „ebensogut im Zoologischen Garten nach dem Leben aufgenommen sind wie unsere anderen Tierbilder" (Heck 1899: 8). In ihrer nachträglichen Umgestaltung orientierte sich die Abbildung damit offensichtlich an den Illustrationen des Thierlebens sowie an den Aufnahmen des ebenfalls im Vorwort erwähnten Ottomar Anschütz. ${ }^{21}$ Während Heck im Vorwort bei den Zeichnungen noch die Verluste „auf dem Wege durch das Auge in den Stift und den Pinsel des Künstlers" beklagt hatte (Heck 1899: 3 f.), blieb der Pinsel nun in der Hand des Retuscheurs - bei der Gestaltung dieser Bilder jedoch unverzichtbares Werkzeug.

Hecks Lebende Bilder stießen bei der Kritik auf Begeisterung. So hob der Zoologe Oskar Boettger (1844-1910) in dem von ihm seit 1896 herausgegebenen Zoologischen Garten den bisher unerreicht großen Maßstab der fotografisch getreu wiedergegebenen Originale hervor sowie ihren unentbehrlichen Wert nicht nur für „Haus und Schule“, sondern „auch für den Zoologen vom Fach und für den gestaltenden Künstler als beste existie- 
rende Abbildungen“ (Boettger 1900). Auch der bekannte Wissenschaftspopularisierer Wilhelm Bölsche (1861-1939) lobte diesen Versuch, der „von höchstem, wissenschaftlichem Wert“ sei: „Mit diesen authentischen Porträts beginnt eine neue Epoche des Tierbildes, vor der alle älteren Bildwerke, selbst die besten, einfach antiquiert sind“ (Bölsche 1900: 150). Die eingangs zitierte Rezension in der Zeitschrift Deutsche Kunst und Dekoration hob sogar den Umstand positiv hervor, dass die Tiere „meist mit landschaftlich sich anpassender Szenerie" ins Bild gesetzt seien (Anonym 1899: 242). Aber gerade diese Eigenschaft der Abbildungen war nun keineswegs neu, sondern seit den Illustrationen des Thierlebens in die Darstellungskonventionen der populären Zoologie eingegangen.

\section{Dramatik im Blitzlicht: Carl Georg Schillings' Tierfotografien aus Ostafrika}

Anschütz' Fotografien erlangten zwar eine große Bekanntheit und Hecks Lebende Bilder stellten einen beachtlichen verlegerischen Erfolg dar, eine Sensation in Deutschland lösten jedoch erst die Aufnahmen freilebender Tiere aus, die der Jäger und Fotograf Carl Georg Schillings (1865-1921) in Afrika gemacht hatte. Schillings hatte zwischen 1896 und 1904 vier Expeditionen nach Deutsch-Ostafrika (heute in etwa Tansania) unternommen und drei davon selbst geleitet. Da sich seine Interessen dabei von der Jagd zur Fotografie verschoben, kann er als prototypisches Beispiel für den engen Zusammenhang von Jagd und früher Tierfotografie gelten. In diesem Sinne hat Bernhard Gissibl (2005) Schillings' Aufnahmen zum einen als Jagdtrophäen und damit als Manifestationen von Männlichkeit gedeutet und zum anderen ihre Legitimationsfunktion für den Kolonialismus betont, da sie den weißen Jäger in seiner Funktion als Naturbezwinger und Kolonialherrn bestätigten. Diese Interpretation ist zwar in sich schlüssig und gut begründet, für den vorliegenden Argumentationszusammenhang erhalten Schillings' Fotografien ihre Bedeutung jedoch primär durch den von Gissibl vernachlässigten Kontext aus zeitgenössischer Tierfotografie, populärer Zoologie und „biologischer Perspektive“.

Schillings' Konversion vom Jäger zum Fotografen vollzog sich in erheblichem Maße unter dem Einfluss Ludwig Hecks, da dieser sich angeblich wünschte, dass Schillings „wenigstens einen Abglanz all dieses imponierenden Naturlebens mit nach Hause bringen" möge (Heck 1905: 4, 1938: 236 f.; Schillings 1905: 24; Becker 2008: 39). Heck stellte deshalb zu Beginn des Jahres 1899 den Kontakt zu dem Militärfotografen Martin Kiesling her, der Schillings nicht nur mit den Grundlagen der Aufnahmetechnik vertraut 
machte, sondern ihn auch mit tropentauglichen Kameras, Objektiven und fotografischen Platten ausrüstete. Im Anschluss entwickelte Kiesling zusammen mit der optischen Anstalt C.P. Goerz außerdem eine mit Drähten zur Selbstauslösung versehene Blitzvorrichtung, die Nachtaufnahmen von Tieren ermöglichen sollte (Kiesling 1901; Schillings 1905: 24-26).

Aus heutiger Perspektive wirkt der überwiegende Teil von Schillings' Aufnahmen zwar ziemlich unspektakulär - Gissibl beurteilt sie als teilweise recht „dürftig“ -, das zeitgenössische Publikum reagierte jedoch mit großer Begeisterung und war offenbar davon fasziniert, lebende Tiere in freier Wildbahn zu sehen und nicht vor bemalten Kulissen oder in den Gehegen der Zoos. Vor diesem Hintergrund wird auch verständlich, warum Schillings immer wieder betonte, auf jede Art von Retuschen verzichtet zu haben, und warum er sich außerdem klar von der angeblich weit verbreiteten Praxis distanzierte, Bilder mit toten oder präparierten Tieren in natürlicher Umgebung aufzunehmen. Selbst dem ansonsten von ihm durchaus geschätzten Ottomar Anschütz unterstellte er aufgrund der wechselnden Umgebungen seiner Raubtieraufnahmen im Breslauer Zoo eine „Täuschung der Leser" (Schillings 1905: 17 f.). Aus dem Verzicht auf jegliche Manipulation leitete Schillings schließlich den besonderen wissenschaftlichen Wert seiner Aufnahmen ab und übernahm als Beleg für ihre Abbildungsqualität in "absoluter dokumentarischer Naturtreue" den von Heck geprägten Begriff der "Natururkunde“ (Schillings 1905: X, 1906: XIII). Dieser Begriff machte in der populären Zoologie schnell Karriere und diente bald zur Kennzeichnung naturkundlicher Aufnahmen mit wissenschaftlichem Anspruch (Schulz 1908/1909; Wandolleck 1911: 50-52).

Den größten Anteil an Schillings' Erfolg hatten aber zweifellos die Nachtaufnahmen, die er von seiner vierten Expedition im Jahr 1903 zurückbrachte. Sie lieferten ihm auch das Stichwort für den ersten Teil seines Buchtitels Mit Blitzlicht und Büchse. Schillings band dazu einzelne Beutetiere wie Esel oder Stiere an Bäumen fest und spannte in einiger Entfernung davon Stolperdrähte, die bei der Berührung durch näherkommende Raubtiere die eigens entwickelte Blitzanlage und den Verschluss der zuvor ausgerichteten Kamera auslösten (Kiesling 1905: 68-71). Die für das damalige Publikum sensationellen Fotos jagender Raubkatzen waren damit das Ergebnis eines aufwendigen Arrangements aus technischen Vorrichtungen und natürlichen Objekten: So schuf das Blitzlicht eine vollkommen künstliche Beleuchtungssituation und erschreckte die Tiere häufig, wie später vereinzelt kritisiert wurde (Wandolleck 1911: 50). Außerdem nahm die Fixierung den Beutetieren jede Chance, dem Angriff zu entkommen. Dennoch konnten die Leser bei einer der Abbildungen (Abb. 6) in der Bildunterschrift lesen, dass sie „eine Szene aus dem Kampf ums Dasein“ vor sich hätten (Schillings 1905: 291). Diese darwinistische Formel sugge- 


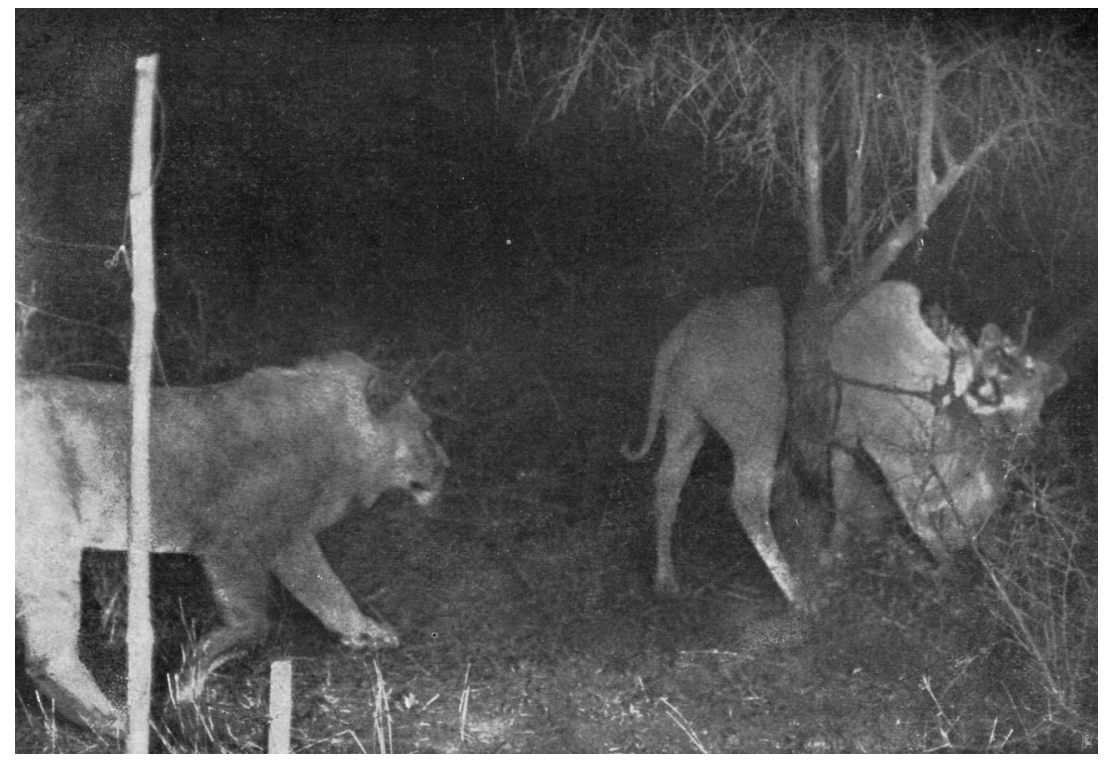

Abb. 6 Schillings Aufnahme vom Angriff zweier Löwen auf einen Stier war das Ergebnis eines aufwendigen Arrangements aus Stolperdrähten, Stativen, Blitzlicht, Kamera und einem fixierten Beutetier (Schillings 1905: 291)

rierte nicht nur die naturgetreue Wiedergabe tierischen Lebens, sondern bediente auch das Bedürfnis des Publikums nach Dramatik und Spannung. Zugleich wird deutlich, dass selbst Schillings, trotz aller Selbstverpflichtungen auf größte Naturtreue, nicht in der Lage war, spektakuläre Tierbilder ohne ein Mindestmaß an Inszenierung herzustellen.

Nach der Rückkehr von seiner letzten Expedition ließ Schillings von vielen seiner belichteten Platten Diapositive anfertigen, die er auf Vortragsveranstaltungen entweder selbst präsentierte oder Ludwig Heck zur Verfügung stellte, der dazu einen eigenen Vortrag über das „Tierleben in der deutsch-ostafrikanischen Wildnis“ ausgearbeitet hatte. Diese Vorträge trugen wesentlich dazu bei, dass die Berliner Urania in den Jahren 1904 und 1905 einen bis dahin unerreichten Besucherrekord zu verzeichnen hatte (Urania Berlin 1913: 37, 45; Heck 1938: 238). Beim Publikum hinterließen die Dias offenbar einen tiefen Eindruck. So ist einer Schilderung Wilhelm Bölsches zu entnehmen, wie die Abfolge der Dias den Eindruck erzeugte, dass sich eine Löwin auf die im Bild zuvor gezeigte Antilopenschar zu stürzen schien, „in hohem Schwung nur mit einer Tatze erst den Boden berührend, mit dreien in der Luft" (Bölsche 1904: 200 f.). Im Zuge dieser Vorträge wurden auch Schillings' Bücher Mit Blitzlicht und Büchse und Der Zauber des Elelescho große Erfolge, die mit Reproduktionen von jeweils über 300 seiner Aufnahmen 1905 und 1906 im Leipziger Voigtlän- 
der Verlag erschienen und innerhalb weniger Jahre mehrere Auflagen und Ausgaben erlebten.

Heck hatte wesentlichen Anteil daran gehabt, dass sich Schillings zum Fotografen ausbilden ließ, er hatte dessen Aufnahmen mit dem Begriff „Natururkunden“ geadelt und sie in Vorträgen selbst präsentiert; man kann deshalb davon ausgehen, dass er Schillings Verständnis von Zoologie erheblich beeinflusste. Denn obwohl Schillings sich ursprünglich wohl primär als Jäger begriffen haben dürfte, zeigte er Interesse an zoologischen Fragen, die die „biologische Perspektive“ widerspiegeln. Zwar nahm die Schilderung seiner Jagderlebnisse den größten Raum ein, aber schon zu Beginn seines ersten Buches widmete er der „Tierpsyche“ ein eigenes Kapitel, das hauptsächlich auf seinen Erfahrungen mit einem lebend gefangenen jungen Nashorn beruhte (Schillings 1905: 28-35; Daum 2002: 433-435). Das wurde durchaus als ernstzunehmender wissenschaftlicher Beitrag anerkannt, wie Oskar Boettgers Rezension im Zoologischen Garten belegt (Boettger 1905: 90). Hinzu kam noch Schillings zunehmender Einsatz für den Naturschutz und die Bewahrung des afrikanischen Tierreichtums (Schillings 1905: 7-16; Becker 2008: 121-152). Denn er stellte damit nicht nur das Leben der Tiere über ihre Bedeutung als Jagdwild und Trophäe, sondern verfolgte auch ganz ähnliche Ziele für Afrika wie einige andere Praktiker der Naturgeschichte für Deutschland.

\section{Heimische Wildtiere in fotografischer Objektivität}

Aufgrund des großen Zulaufs zu Schillings' beziehungsweise Hecks Vorträgen war es für den Voigtländer Verlag nicht schwer, den Erfolg der Bücher vorherzusehen. Sowohl einige Rezensenten als auch Heck und Schillings hatten auf die paradoxe Situation hingewiesen, dass mit den Aufnahmen nun „Natururkunden“ einer exotischen Tierwelt vorlägen, während für die heimischen Wildtiere Vergleichbares noch fehle (Schillings 1905: 21; Heck 1905: 4 f.; Heinroth 1905: 60). Der Verlag hätte sein Geschäft also schlecht verstanden, wenn er nicht umgehend mit den Vorbereitungen für ein vergleichbares Werk zur mitteleuropäischen Fauna begonnen hätte. Um die dazu notwendigen Fotografien zu beschaffen, schrieb der Verlag bereits Anfang des Jahres 1905 Preise für die besten eingesandten Tieraufnahmen aus und stellte dafür insgesamt 3.000 Mark in Aussicht. Ausdrücklich von der Teilnahme ausgeschlossen waren Bilder von zahmen oder gefangen gehaltenen Tieren sowie von Haustieren, auch jede Retusche an Negativ oder Abzug war untersagt (R. Voigtländers Verlag 1905; Anonym 1905). 
Im Zuge dieses Preisausschreibens beauftragte der Voigtländer Verlag Schillings' fotografischen Lehrer Martin Kiesling, eine Anleitung zum Photographieren freilebender Tiere zu verfassen, um den Teilnehmern „die Arbeit zu erleichtern" (Kiesling 1905: 7 f.). Da sich Kiesling aber vorwiegend auf technische Hinweise beschränkte, stand sie bald im Schatten der Photographischen Naturstudien, die im gleichen Jahr erschienen und aus der Feder des Braunschweiger Museumsassistenten, Jägers, Naturkundlers und Fotografen Hermann Meerwarth (1870-1943) stammten (Ahrens 2004: 99). ${ }^{22}$ Mit dem Buch richtete er sich einerseits an Amateurfotografen (im Unterschied zum Berufsfotografen und zum „Knipser“) und andererseits an Naturkundler und Jäger, um ihnen jene Kenntnisse zu vermitteln, die zur erfolgreichen Tierfotografie noch fehlten. Meerwart behandelte deshalb nicht nur die fotografische Technik, sondern sehr viel stärker als Kiesling auch die Situationen, in denen Tier und Fotograf vor der Kamera interagieren.

Sowohl Meerwarth als auch Kiesling verbanden mit ihren Anleitungen den Anspruch, Aufnahmen zu ermöglichen, die der Wissenschaft dienen, und beide postulierten dabei die Überlegenheit der „objektiven“ Fotografie über die „subjektive“ Zeichnung (Kiesling 1905: 9-12, 76). Bei Meerwarth hieß es dazu explizit, dass „die Camera und die Platte [...] nach physikalischen und chemischen Gesetzen ,objektiv" " arbeiten würden und ihre Bilder „deshalb eine zwingende Überzeugungskraft für ihre Naturtreue“ besäßen. „Der Künstler dagegen kann nur ,subjektiv‘, nach individueller Veranlagung schaffen, er muß es sogar und kann deshalb eben des Naturforschers Zweifel an der absoluten Naturwahrheit seiner Schöpfungen nie ganz beheben“ (Meerwarth 1905: 1). Ein derartiger Anspruch auf Objektivität implizierte eigentlich, dass sich der Fotograf möglichst jedes Eingriffs in das Aufnahmeverfahren zu enthalten hätte (Daston \& Galison 2007: 121-200). Retuschen standen für Meerwarth deshalb gar nicht mehr zur Debatte, sondern galten ihm als unzulässige Manipulation. Außerdem kritisierte er Aufnahmen von präparierten Tieren oder von Haustieren in der freien Natur, wenn diese nicht entsprechend gekennzeichnet waren.

Trotz seines expliziten Anspruchs auf Objektivität erteilte Meerwarth seinen Lesern zahlreiche Ratschläge, um die Situation vor der Kamera zu beeinflussen. Bei der Aufnahme von Ameisenhaufen erklärte er beispielsweise, dass man hier die Möglichkeit hätte, „ohne der Naturwahrheit zu nahe zu treten, etwas nach[zu]helfen, indem man irgend ein fremdes Tier auf den Bau wirft oder die Ameisen durch Beunruhigung des Baues zum Wegschleppen ihrer Puppen veranlaßt" (Meerwarth 1905: 54). Meerwarth war sich also durchaus bewusst, dass sich die Zulässigkeit des von ihm vorgeschlagenen Eingriffs nicht von selbst verstand, er hielt diesen aber für nicht so gravierend, um dafür auf zusätzliche Dramatik in der Bildgestaltung zu 
verzichten. Seine Ratschläge schlossen auch gezielte Veränderungen der vorgefundenen Umgebung mit ein, wenn sich dadurch die Attraktivität von Fotos, etwa bei Vogelnestern, steigern ließ. Zur Vermeidung irritierender Lichtflecken empfahl er beispielsweise in einigem Abstand vom Nest ein großes lichtgraues Tuch aufzuspannen, störende Zweige abzuschneiden oder wegzubiegen, niemals jedoch abzubrechen. Noch einen Schritt weiter ging sein Rat, auch „etwas ,Kunst' ruhig mithelfen“ zu lassen: „Ein paar Blumen, die einige Meter vom Nest entfernt stehen, eine blühende Brombeerranke z. B. mögen wir immerhin abschneiden und geschickt an einem im Brombeerbusch stehenden Nest anbringen - wir erhöhen den Bildwert unserer Aufnahme, ohne Unnatürliches hinzugebracht zu haben" (Meerwarth 1905: 78-82). Als fotografische Inszenierung ${ }^{23}$ kann man schließlich seinen Vorschlag werten, noch flugunfähige Vogeljungen aus ihrem Nest zu nehmen und sie nebeneinander auf einen zuvor präparierten Ast zu setzen. Um scharfe Fotos von der Fütterung einzufangen, bräuchte man dann nur noch auf die Rückkehr der Eltern zu warten (Meerwarth 1905: $90 \mathrm{f}$.).

Meerwarths Naturstudien wurden durchweg positiv besprochen, dabei störte sich keiner der Rezensenten an seinen Empfehlungen, in den Bildaufbau einzugreifen oder Aufnahmen zu arrangieren. Kritik rief noch am ehesten seine Forderung hervor, für die Naturforschung und -vermittlung grundsätzlich der „Camera das Recht vor dem Pinsel“ einzuräumen, da einige Rezensenten nicht daran glaubten, ganz auf die Zeichnung verzichten zu können. Sie sahen den Wert der mit Hilfe von Meerwarts Ratgeber entstandenen Aufnahmen deshalb auch in ihrer Funktion als Zeichenvorlage (Meerwarth 1905: 2; Cahn 1906; Hanstein 1906).

Wie der Kontakt zwischen Meerwarth und dem Voigtländer Verlag zustande kam, lässt sich nicht mehr rekonstruieren, aber zweifellos war Meerwarth bestens qualifiziert, das geplante, mit Fotografien illustrierte Werk über die heimische Fauna herauszugeben. Unter dem Titel Lebensbilder aus der Tierwelt erschien es in mehreren Bänden zwischen 1908 und 1912. Zu den ersten und wichtigsten Mitarbeitern, die Meerwarth gewinnen konnte, gehörte der schon recht bekannte Schriftsteller und Journalist Hermann Löns (1866-1914), der auch einige Semester Zoologie studiert hatte (Deimann 1927; Dupke 1994: 39-41). Neben Schriftstellerei und Journalismus, die ihm den Lebensunterhalt sicherten, arbeitete Löns in den Jahren um 1905 auch an einer Studie zur "Wirbeltierfauna der Provinz Hannover“, für die er im Zoologischen Garten mit einem „Aufruf“ um Unterstützung bat (Löns 1905). Weitere Mitarbeiter für die Lebensbilder fand Meerwarth ebenfalls unter den Autoren der Zeitschrift, die mit ihrer „biologischen Perspektive“ auf die Tiere, mit ihrem Interesse an deren Lebensweise, Verhalten oder "Psychologie“, seine eigene wissenschaftliche Ausrichtung teil- 
ten. ${ }^{24} \mathrm{Zu}$ ihnen gehörte etwa das Maler- und Schriftstellerehepaar Karl (*1877) und Else Soffel (1877-1968), die ihre Publikationstätigkeit für den Zoologischen Garten nicht nur mit mehreren Beiträgen in den Lebensbildern fortsetzten; ${ }^{25}$ ab dem zweiten Band übernahm Karl Soffel auch die Mitherausgeberschaft.

Als Praktiker der Naturgeschichte betonte Meerwarth im Vorwort den Unterschied der Lebensbilder zur „Mehrzahl naturwissenschaftlicher Bücher“, da sie sich auf eine „biologische Darstellung des Tieres“ beschränkten und auf „systematische Beschreibungen“ verzichteten: Erstrebt sei „lediglich die Schilderung des Lebens“ (Meerwarth 1909: VII f.). Ein Verzicht auf Systematik charakterisierte auch die zoologische Gliederung der Bände, die nur einer groben Einteilung nach Säugetieren, Vögeln, Amphibien usw. folgten. Für jede weitere Systematisierung innerhalb der einzelnen Bände wies der verfügbare fotografische Bildbestand nämlich zu große Lücken auf, obwohl die Aufnahmen des Preisausschreibens durch gezielte Ankäufe von deutschen, englischen und amerikanischen Fotografen ergänzt worden waren.

Statt durch zoologische Systematik zeichnete sich das Werk durch „die Naturwahrheit des Lichtbildes“ aus, das Meerwarth erneut "als das einzig vollkommen befriedigende Illustrationsmittel“ für naturwissenschaftliche Bücher propagierte (Meerwarth 1909: V, Hervorh. i. Orig.). Damit versuchte er offenbar, dem Buchprojekt als Ganzem durch die behauptete Objektivität der Kamera und ihrer Bilder eine möglichst weitgehende wissenschaftliche Reputation zu sichern. Obschon sich seit der Jahrhundertwende die Opposition zwischen den systematisch arbeitenden Universitätszoologen und den Praktikern der Naturgeschichte abgeschwächt und die "biologische Perspektive“ auch akademische Anerkennung gefunden hatte, wirkten hier offenbar die Auseinandersetzungen der vorangegangenen Jahrzehnte nach (Nyhart 2009: 355). Außerdem fehlte den von Meerwarth versammelten Autoren und Fotografen in den meisten Fällen eine institutionelle Heimat, die sie als professionelle Wissenschaftler ausgewiesen hätte. ${ }^{26}$ Als Amateure waren sie deshalb vermutlich besonders empfänglich für die Rhetorik der fotografischen Objektivität, da sie half, den wissenschaftlichen Wert ihrer Naturbeobachtungen zu steigern. ${ }^{27}$

Die Lebensbilder waren zwar als Tierbuch mit wissenschaftlichem Anspruch konzipiert, doch richteten sie sich an ein breites Publikum, das mindestens so sehr an attraktiven wie an objektiven Bildern interessiert war. Meerwarth rechtfertigte deshalb die Entscheidung, das Werk ausschließlich mit Fotografien zu illustrieren, auch in ästhetischer Hinsicht und hob unter anderem deren Vorzüge bei der Darstellung „zartester Körperformen“ gegenüber der Zeichnung hervor (Meerwarth 1909: VI). Tatsächlich waren Quantität und Qualität der versammelten Fotos nach zeitgenössi- 
schen Maßstäben außergewöhnlich. Soweit sich das ohne weitere Angaben beurteilen lässt, erfüllten die meisten Fotografien auch die eigenen Ansprüche und zeigten wildlebende Tiere in freier Natur. Nur die Aufnahmen kleinerer Tiere stammten teilweise aus Vivarien; sie waren in der Regel aber so arrangiert, dass die Künstlichkeit der Umgebung nicht in Erscheinung tritt und diese Information nur der Bildunterschrift zu entnehmen ist. Im Vergleich zu Schillings' Fotos aus Ostafrika fehlten den Lebensbildern zwar so spektakuläre Szenen wie dessen nächtliche Raubtierangriffe, ansonsten waren die Aufnahmen aber überwiegend schärfer und kontrastreicher, die Tiere erschienen meist größer und ihre Darstellung war vielfältiger.

Noch stärker als der Verlag, der die ersten Bände der Reihe mehrfach nachdrucken konnte, dürften Meerwarth und seine Mitstreiter die Publikation der Lebensbilder als Erfolg verbucht haben. Denn während ihnen zuvor kaum etwas Anderes übriggeblieben war, als ihre schriftlichen oder fotografischen Naturbeobachtungen einzeln im Zoologischen Garten oder in fotografischen Fachzeitschriften zu veröffentlichen, wurden diese nun $\mathrm{zu}$ einem Gesamtwerk vereint und der wissenschaftlichen wie der breiten Öffentlichkeit zugänglich gemacht. Das verschaffte dem Werk nicht nur Anerkennung aus der universitären Zoologie, der Dresdner Professor Benno Wandolleck (1864-1930) begriff die „biologische Photographie“ sogar grundsätzlich als eine Möglichkeit für den Laien, der Wissenschaft zu nützen und die vorherrschenden Ansichten vom Leben der Tiere zu verändern (Wandolleck 1911: 49-52). Die Resonanz in der Presse reichte von der populären Berliner Illustrirten Zeitung, die ausführlich Notiz nahm, ${ }^{28}$ bis hin zur katholischen Kulturzeitschrift Hochland, in der der Philosoph Max Ettlinger (1877-1929) „das mustergültige Werk“ mit seinen „prächtigen Bildern“ lobte und als Vorbild für die „Wiederbelebung biologischer Beobachtungsfreude" anführte (Ettlinger 1910). ${ }^{29}$ Auf besonderes Interesse stießen die Lebensbilder auch bei etlichen Museumsmitarbeitern, die sie als „Musterbuch“ für die Präparation und - in Leipzig und Altona - als Vorlage für biologische Gruppen und Dioramen nutzten (Köstering 2003: 171; Ahrens 2004: 132).

\section{Fazit}

Seit Mitte des 19. Jahrhunderts war die populäre Zoologie in Deutschland stark von der „biologischen Perspektive“ geprägt. Die Änderungen, die sich daraus für die Darstellung von Tieren ergaben, hat Nyhart (2009) detailliert am Beispiel von Philipp Leopold Martins (1815-1886) unermüdlichem Engagement für einen lebensechten Präparations- und Präsentati- 
onsstil ausgeführt sowie an der um die Jahrhundertwende kulminierenden Auseinandersetzung über die museale Aufstellung dramatischer biologischer Gruppen. Die vorangegangene Analyse liefert nun gute Argumente dafür, dass sich diese Veränderungen hin zu Lebendigkeit und Dramatik in der populären Tierzeichnung etwa zeitgleich vollzogen oder teilweise vorweggenommen wurden. So bezogen die Zeichner den Lebensraum eines Tieres schon seit Brehms Thierleben in ihre Illustrationen ein, weil er mit der „biologischen Perspektive“ an Relevanz gewonnen hatte. (Die frühere Funktion, eigene Augenzeugenschaft nachzuweisen, besaß demgegenüber nur noch geringe Bedeutung). Die bildtechnischen Bedingungen der Zeichnung erlaubten es den Illustratoren dabei problemlos, ein einheitliches Bild aus verschiedenen Quellen zu entwerfen, während das bei Dioramen oder Fotografien aus unterschiedlichen Gründen viel aufwendiger war. Zudem bot das populärwissenschaftliche Feld größere Spielräume als die Fachwissenschaft, um diese bildtechnischen Freiheiten auszuschöpfen und etwa Landschaften ins Bild zu integrieren, die ganz oder teilweise auf der eigenen Imagination beruhten. Der Erfolg dieser Art der Illustration wäre jedoch kaum denkbar gewesen, wenn sie nicht auch auf der Nachfrageseite, also letztlich beim breiten Publikum, auf regen Zuspruch gestoßen wäre; Redaktionen und Verlage nutzten lebensvolle oder dramatische Tierillustrationen jedenfalls gerne als Blickfang oder Verkaufsargument und versprachen sich davon höhere Absatzzahlen.

Schon bald nach der Erfindung der Fotografie standen Malerei und Zeichnung mit ihr in intensivem Austausch. Bekannte und weniger bekannte Künstler nutzten Fotografien für ihre Arbeit als Vorlage, Studienmaterial und Gedächtnisstütze (Pohlmann 2004a). In diesem Sinne wurden auch Anschütz', Hecks oder Meerwarths Tierfotografien von den Fotografen selbst ebenso wie von Journalisten oder Zoologen als willkommenes Hilfsmittel für Zeichnungen in Kunst oder Wissenschaft verstanden. Im Gegenzug nahmen sich Fotografen von Beginn an die Malerei und ihre Ästhetik als Vorbild, um den Kunstwert ihrer Bilder zu erhöhen und ihnen damit kulturelle Anerkennung zu verschaffen. Sie standen hier aber vor der Aufgabe, die Natur nicht mechanisch zu reproduzieren, sondern zumindest im fertigen Bild ihre eigene Individualität und subjektive Interpretation zum Ausdruck zu bringen (Kaufhold 1986: 40-43; Daston \& Galison 2007: 140). Ein ambivalentes Verhältnis zeigte sich dagegen in den Bildern, die Anschütz sowie Heck und sein Retuscheur angefertigt hatten: Denn sie gestalteten ihre Aufnahmen nach dem Vorbild von Zeichnungen zwar bewusst mit Kulissen oder Retuschen, nahmen aber dennoch wie selbstverständlich fotografische Naturtreue für sie in Anspruch. In ähnlicher Weise schrieben auch Schillings, Kiesling und Meerwarth ihren Aufnahmen „Urkundcharakter" beziehungsweise „Objektivität" zu und 
grenzten sie dafür von der Subjektivität der Zeichnung ab. Darüber hinaus behaupteten sie aber noch, Aufnahmen anzufertigen, die von äußeren Eingriffen in den fotografischen Prozess frei seien, und leiteten daraus ihren besonderen Wert für die Wissenschaft ab. Obschon diese Ansprüche in Theorie und Praxis immer wieder unterlaufen wurden, wird hier deutlich, dass sich das Ideal der „mechanischen Objektivität“ nicht nur auf die von Daston und Galison (2007) untersuchten fachwissenschaftlichen Diskurse und Bilderpraktiken beschränkte, sondern auch für Wissenschaftsvermittler, Amateurwissenschaftler und eine interessierte Öffentlichkeit Gültigkeit besaß. Trotz vereinzelter Kritik dominierte also auch in diesem Feld der Glaube an die objektivierende Kraft der Kamera.

Bei der Lektüre zentraler Schriften zur naturkundlichen Fotografie (Kiesling 1905; Meerwarth 1905; Wandolleck 1911) entsteht so der Eindruck, dass engagierte Amateure mit dem Besitz einer Kamera die Chance erhielten, ihre Beobachtungen wild lebender Tiere in wissenschaftlich wertvollen Bildern zu dokumentieren und dadurch ihre Position im naturkundlichen Diskurs zu verbessern. Das wäre etwa mit den erweiterten Möglichkeiten vergleichbar, die sich Amateuren seit Mitte des 19. Jahrhunderts durch die Gründung zahlreicher Zoos boten: Wie Hochadel (2011) herausgearbeitet hat, konnten hier nämlich die unterschiedlichsten Personengruppen teilweise über längere Zeiträume hinweg Tiere beobachten und dann mit anderen Interessierten darüber in Zeitschriften wie dem Zoologischen Garten in einen - durchaus auch wissenschaftlichen - Austausch treten. Inwieweit die Kamera die Praktiken der Amateure tatsächlich veränderte, ist bislang allerdings schwer zu sagen und bedürfte weiterer Forschungen. Naturkundler wie Meerwarth (1905: 6) oder Hanstein (1906) hätten es wohl schon als Erfolg verbucht, wenn Tiere, Nester und Eier seltener gejagt beziehungsweise gesammelt und stattdessen vermehrt fotografiert worden wären. Große Pläne schmiedete hingegen der Lehrer Georg Schulz (1908: 5), der alle interessierten Amateure aufforderte, seltene Naturobjekte in fotografischen Urkunden festzuhalten, um diese dann einem Museum, einem wissenschaftlichen Institut oder einer anderen noch $\mathrm{zu}$ gründenden staatlichen Stelle zur dauerhaften Archivierung $\mathrm{zu}$ überantworten. Immerhin existieren einige Beispiele dafür, dass zumindest einzelne Amateure ihre Kamera in dieser Weise nutzten und durch eigene Publikationen auf sich aufmerksam machten (Bartels 1910) oder, motiviert von den Preisen des Voigtländer Verlags, mit ihren Aufnahmen zum Gelingen von Meerwarths Lebensbildern aus der Tierwelt beitrugen. 


\section{Danksagung}

Der Artikel basiert auf Forschungen zu meinem Projekt „Dramatik, Darwinismus und der Kampf um die Aufmerksamkeit des Lesers. Zoologische Illustrationen in deutschen Publikumszeitschriften um 1900", für dessen Förderung mein Dank der DFG gilt. Für Anregungen, Hinweise und Korrekturvorschläge möchte ich mich herzlich bei Kärin Nickelsen, Cornelia Kemp, Thomas Wieland, Helmuth Trischler, Ulf Hashagen, Mareike Vennen, Peter Schüßler und Anna Falkenberg bedanken. Ein besonderer Dank geht auch an die beiden Gutachter bzw. Gutachterinnen, die mit ihren Empfehlungen insbesondere bei der Überarbeitung des Fazits geholfen haben.

\section{Anmerkungen}

1 Als wichtigste Ausnahme wären die Arbeiten zu Ernst Haeckels Kunstformen der Natur zu nennen (z. B. Gebauer 2013; Kockerbeck 1986).

2 Ohne Berücksichtigung blieben damit nur einige Zeitschriftenartikel, thematisch außerdem Publikationen zu Fischen, Amphibien, Reptilien und Insekten.

3 Mangelndes Talent oder die Möglichkeit zu Zeit- und Kostenersparnis spielten beim Kopieren dagegen nur eine untergeordnete Rolle (vgl. Nickelsen 2007: 68).

4 Dass dabei Kretschmers Zeichnung eines Zwergbocks, auf der das zierliche Wesen nach Brehms Einschätzung „plump und groß“ erschien, keinen Eingang ins Thierleben fand, war freilich nur konsequent (Brehm 1863b: 325, 327).

5 Die spärliche Ausbeute an Tierzeichnungen erklärt sich vermutlich durch Kretschmers Auftrag, primär Reisegesellschaft und Jagdaktivitäten sowie Land und Leute darzustellen (Anonym 1862, 1864).

6 Vgl. dazu Mey (1989: 2, Tabelle der Zeichner) und Gall (2011: 108).

7 Für die Übersendung eines Einzelheft-Titelblattes in Kopie danke ich herzlich Herrn Dr. Eberhard Mey.

8 Allerdings weist Brehm (1865a: 746) nur bei der Abbildung einer Warzenschweinart explizit darauf hin, dass sie „leider dem lebenden Thiere nicht“ entspreche.

9 Für eine unsignierte und eine von Kretschmer signierte Zeichnung gibt Brehm (1864: 220, 590) jeweils Zeichnungen Joseph Wolfs als Vorlage an, für die von Zimmermann signierte Abbildung eines Schuppentiers lieferte eine Zeichnung von Brehms Freund Theodor von Heuglin (1824-1876) die Vorlage (Brehm 1865a: 311, 316).

10 Es fehlt hier der Raum, um Brehms Übernahmen im Detail zu diskutieren. Erste Hinweise zu Woods Vorbildfunktion für Brehm finden sich bei Schneider (1991: 63), Schlawe (1994: 39) und Haemmerlein (2015: 69 f.); zu Wood vgl. Lightman 2007: 169-196.

11 Hinweise auf die Benutzung von Vorlagen gibt Brehm $(1864: 473,812)$ z. B. für zwei Abbildungen Kretschmers (1. Bd., S. 474, 2. Bd., S. 808); ohne Hinweis bleibt Kretschmers Orientierung an Illustrationen von Wood $(1859: 527,541,545)$ beispielsweise bei seinen Abbildungen im zweiten Band auf den S. 836 (spiegelverkehrt), 850 (spiegelverkehrt), 867 (spiegelverkehrt). 
12 Vogts Besprechung war in Auszügen zusammen mit weiteren „Stimmen der Presse" vom Verlag an das Ende des zweiten Säugetierbandes angehängt worden. URL: http://reader.digitale-sammlungen.de/de/fs1/object/display/bsb10707136_00956.html (Abruf am 21.03.2016).

13 Nach den von mir - ursprünglich für andere Zwecke - erhobenen Daten erfolgte eine derartige Trennung bei der Zoologie zwischen 1890 und 1905 in 116 Fällen (27 \% gemessen an der Gesamtzahl der zum Thema erfassten Beiträge), bei der Schifffahrt in 21 Fällen (15\%), bei der Botanik in 10 Fällen (6\%) und bei allen weiteren Themen seltener als zehnmal. Allerdings wurden die für die Berechnung notwenigen Seitenzahlen der Abbildungen ursprünglich aus rein praktischen Erwägungen in die Datensätze aufgenommen, die deshalb vermutlich unvollständig sind; es dürfte dabei aber nicht zu systematischen Fehlern gekommen sein.

14 Diese Argumentation lehnt sich an Methoden der Kommunikationswissenschaft an, die Artikel und Abbildungen u. a. hinsichtlich ihrer Position und Größe innerhalb einer Publikation bewerten, vgl. Wilke (1984), Staab (1990).

15 In seinem Nachruf stand zwar, dass „Specht auf seinen weiten Reisen [...] die Tierseele genau studiert" habe, doch trotz vieler biografischer Details fand nicht eine einzige konkrete Reise als Beleg dafür Erwähnung, Brönnle (1910: 37).

16 So gehen unter den über 500 zoologischen Beiträgen, die zwischen 1880 und 1905 in der Gartenlaube erschienen, überhaupt nur zwölf auf die Theorien Darwins oder evolutionsbiologische Vorstellungen ein.

17 Wenig spricht deshalb für Kösterings (2003: 170, Fn. 66) Annahme, dass Anschütz für diese Anordnung naturkundliche Dioramen als Vorbild dienten. Außerdem setzten sich, wie Köstering selbst ausführt, Dioramen als Gestaltungselemente in den deutschen Naturkundemuseen erst in den 1890er Jahren und damit nach der Entstehungszeit von Anschütz' Aufnahmen durch, vgl. auch Gall (2016: 86).

18 Es ist deshalb kein Zufall, dass die Universität der Künste in Berlin mit rund 1.300 Aufnahmen die wohl umfangreichste Sammlung von Anschütz' Fotografien besitzt. Nach Auskunft des Archivleiters Dr. Dietmar Schenk stammen sie aus den Beständen der Königlichen Akademie der Künste zu Berlin und der Unterrichtsanstalt des Kunstgewerbe-Museums.

19 Vgl. z. B. Neue Beiträge zur Naturgeschichte der Waldhühner, in: Der Zoologische Garten 41 (1900), S. 47; Schutzmittel gegen den Angriff von Raubtieren für Wärter in zoologischen Gärten, in: ebd. S. 187.

20 Anzeige des Werner Verlags. Börsenblatt für den deutschen Buchhandel Nr. 281, 4. Dezember 1899, S. 9304.

21 Vgl. mit ähnlichen Retuschen und ähnlicher Bildunterschrift die Abbildung einer Mufflon-Familie, Heck (1899: 31).

22 Auch in diesem Fall ging die Initiative offenbar vom Verlag aus, der sich eine Umarbeitung von Arthur R. Dugmores Camera and Countryside für die deutschen Verhältnisse wünschte. Als Grund dafür darf man ebenfalls das Preisausschreiben des Voigtländer Verlages vermuten, da Meerwarth im Vorwort direkt Bezug darauf nahm (Meerwarth 1905: III, 7). Durch eigenständige Ergänzungen war sein Buch dann deutlich umfangreicher als die Vorlage.

23 Zur unübersichtlichen Begrifflichkeit vgl. Janecke (2010).

24 So hatte der Pädagoge und Heimatkundler Hugo Otto (1875-1949) allein im Zoologischen Garten 48 (1907) 13 meist kurze Beiträge veröffentlicht. Im ersten Band der Lebensbilder verfasste er die Aufsätze zu Iltis und Wiesel. 
25 Zu den Beiträgen von Karl Soffel vgl. Der Zoologische Garten 46 (1905): Kohlmeise und Resorcinkristalle, 23; Der Zoologische Garten 47 (1906): Waldmaus, 56-58; Fortpflanzung von Planorbis corneus, 84 f.; Atemgeräusche bei Schnecken?, 85; Kleintierleben im Winter, 113-116; Winterkleid des Hermelins, 121; Blaufuchs-Züchtereien in Norwegen, 152 f.; Der Zoologische Garten 48 (1907): Weinbergschnecken, 320; Der Bergmolch, 320 f.; Gewölle der Rabenkrähe, 321; Der Gelbrand, 321; Räubereien der Rabenkrähe, 353; Gefräßigkeit des Wasserfrosches, 353; Materialien zur Psychologie der Tiere: Dohle und Krähe, 361-363.

26 Eine aufwendige Werbebroschüre für die Buchreihe enthält auch eine Liste mit Namen und Berufsangaben der Fotografen, die vor allem aus dem Bildungsbürgertum stammten. Einen professionellen Hintergrund besaßen neben den Berufsfotografen am ehesten drei Mitarbeiter von Forstverwaltungen und ein Präparator, vgl. Meerwarth (1908: 8).

27 Ähnlich auch bei Schulz (1908: 4 f.), der aber statt „Objektivität“ Hecks Wortschöpfung „Urkundtreue“ verwendet.

28 Mit der Kamera durch Wald und Flur. Momentbilder aus dem Leben der Tiere. Berliner Illustrirte Zeitung 15. Mai 1910, 373-375.

29 Umso schwieriger ist die Frage zu beantworten, warum sich der Verlag nicht mehr dazu entschließen konnte, die ursprünglich geplanten Bände über Amphibien, Reptilien und Fische sowie über Insekten zu veröffentlichen, obwohl etwa Löns' Beitrag zur Libelle bereits fertiggestellt war (Deimann 1927: 114) und andernorts durchaus gelungene Beispiele für Insektenaufnahmen existierten (Bade 1904; Bartels 1910).

\section{Literatur}

Ahrens, Sabine 2004. 250 Jahre Naturhistorisches Museum in Braunschweig. Eine „Pflanzstätte für die naturgeschichtliche Bildung“. Braunschweig: Staatl. Naturhistor. Museum.

Anonym 1856. Wüsten-Bilder. I. Die Sahara und das Kamel. Die Gartenlaube (23): 301-304.

Anonym 1862. Ein Pirschgang auf Elephanten in den östlichen Hängen des Felsengebirges von Abyssinien. Bruchstücke aus einem Briefe Sr. Hoheit des Herzogs Ernst von Coburg an einen Bekannten. Die Gartenlaube (32): 500-503.

Anonym 1863a. [Besprechung] A. E. Brehm, Dr. Illustrirtes Thierleben. Eine Allgemeine Kunde des Thierreichs. Der Zoologische Garten (4): 113-115.

Anonym 1863b. [Rezension] Illustrirtes Thierleben. Eine Allgemeine Kunde des Thierreichs von Dr. A. E. Brehm. Naturwissenschaftliches Literaturblatt. Beilage zur „Natur“ (12), 2: $10-12$.

Anonym 1864. Reise des Herzogs Ernst von Sachsen-Coburg-Gotha nach Ägypten und den Ländern der Habab, Mensa und Bogos. Leipzig: Arnold.

Anonym 1883. Des Tigers Beute. Die Gartenlaube (42): 681-692.

Anonym 1897. Ein Werk über die mitteleuropäischen Jagdtiere. Photographische Rundschau (11): 156 .

Anonym 1899. Bücherschau. Lebende Bilder aus dem Reiche der Tiere. Deutsche Kunst und Dekoration (5): 240-242.

Anonym 1905. Natur-Urkunden über unsere heimische Tierwelt. Photographische Rund$\operatorname{schau}(19): 98$.

Anschütz, Ottomar 1889. Einige allgemeine Erläuterungen über die Aufnahme wilder Tiere. Photographische Mitteilungen (25), 386: 307-308, Beilage No. 14.

Artinger, Kai 1995. Von der Tierbude zum Turm der blauen Pferde. Die künstlerische Wahrnehmung der wilden Tiere im Zeitalter der zoologischen Gärten. Berlin: Reimer.

Bade, Ernst 1904. Die Tarnkappe der Insekten. Die Gartenlaube (6): 168-169.

Bartels, C. O. 1910. Auffrischer Tat. Beobachtungen aus der niederen Tierwelt in Bilderserien nach Natur-Aufnahmen. Stuttgart: Schweizerbart. 
Becker, Manfred 2008. Bwana Simba - der Herr der Löwen. Carl Georg Schillings. Forscher und Naturschützer in Deutsch-Ostafrika. Düren: Hahne \& Schloemer.

Beetham, Margaret 1990. Towards a Theory of the Periodical as a Publishing Genre. In: Laurel Brake, Aled Jones und Lionel Madden (Hg.). Investigating Victorian Journalism. New York: St. Martin's Press: 19-32.

Blum, Ann S. 1993. Picturing Nature. American Nineteenth Century Zoological Illustration. Princeton/NJ: Princeton University Press.

Boettger, Oskar 1900. Dr. L. Heck, Lebende Bilder aus dem Reiche der Tiere. Werner Verlag, Berlin, 1900. Fol. Lief. 3-16. Der Zoologische Garten (41): 188.

Boettger, Oskar 1905. C. G. Schillings, Mit Blitzlicht und Büchse. Neue Beobachtungen und Erlebnisse in der Wildnis inmitten der Tierwelt von Äquatorial-Ostafrika. Leipzig R. Voigtländers Verlag, 1905. Der Zoologische Garten (46): 89-91.

Bolle, Carl 1863. Illustrirtes Thierleben. Eine allgemeine Kunde des Thierreichs von Dr. A. E. Brehm. Journal für Ornithologie (11), 2: 135-136.

Bölsche, Wilhelm 1900. Lebende Tiere. Mutter Erde (3): 148-151.

Bölsche, Wilhelm 1904. Weltblick. Gedanken zu Natur und Kunst. Dresden: Reißner.

Braun, Marta 1992. Picturing Time. The Work of Etienne-Jules Marey (1830-1904). Chicago: University of Chicago Press.

Braun, Marta 2010. Animal Locomotion. In: Philip Brookman (Hg.). Helios, Eadweard Muybridge in a Time of Change. Göttingen, Washington/DC: Steidl: 271-284.

Brehm, Alfred E. 1855. Reiseskizzen aus Nord-Ost-Afrika oder den unter egyptischer Herrschaft stehenden Ländern Egypten, Nubien, Sennahr, Rosseeres und Kordofahn gesammelt auf seinen in den Jahren 1847 bis 1852 unternommenen Reisen. 3 Bde., Jena: Mauke.

Brehm, Alfred E. 1861a. Aus dem Norden. II Die Vogelberge. Die Gartenlaube (1): 12-15.

Brehm, Alfred E. 1861b. Das Leben der Vögel. Dargestellt für Haus und Familie. Prachtausgabe mit 24 Abbildungen und drei Tafeln in Farbendruck. Golgau: Flemming.

Brehm, Alfred E. 1862. Das kleinste Säugethier. Die Gartenlaube (37): 588-590.

Brehm, Alfred E. 1863a. Ergebnisse einer Reise nach Habesch im Gefolge Seiner Hoheit des regierenden Herzogs von Sachsen-Koburg-Gotha Ernst II. Hamburg: Meißner.

Brehm, Alfred E. 1863b. Vierzehn Tage in Mensa (I, II, III). Globus. Illustrierte Zeitschrift für Länder- und Völkerkunde (3): 161-171, 289-298, 321-332.

Brehm, Alfred E. 1864. Erste Abtheilung. Die Säugethiere. Erste Hälfte. Affen und Halbaffen, Flatterthiere und Raubthiere. In: Alfred E. Brehm, Illustrirtes Thierleben. Eine allgemeine Kunde des Thierreichs, Bd. 1. Hildburghausen: Bibliogr. Inst.

Brehm, Alfred E. 1865a. Erste Abtheilung. Die Säugethiere. Zweite Hälfte. Beutelthiere und Nager. Zahnarme, Hufthiere und Seesäugethiere. In: Alfred E. Brehm, Illustrirtes Thierleben. Eine allgemeine Kunde des Thierreichs, Bd. 2. Hildburghausen: Bibliogr. Inst.

Brehm, Alfred E. 1865b. Vorwort. In: Reinhold Brehm und Theodor F. Zimmermann, Bilder und Skizzen aus dem Zoologischen Garten zu Hamburg. Hamburg: Lührsen: V-VI.

Brehm, Alfred E. 1876. Erste Abtheilung, Säugethiere. Erster Band: Affen und Halbaffen, Flatterthiere, Raubthiere. In: Alfred E. Brehm, Brehms Thierleben. Allgemeine Kunde des Thierreichs. 2. umgearbeitete und vermehrte Auflage, Leipzig: Bibliogr. Inst.

Brehm, Alfred E. und Emil A. Roßmäßler 1864. Die Wirbeltiere des Waldes. In: Alfred E. Brehm und Emil A. Roßmäßler, Die Thiere des Waldes, Bd. 1. Leipzig, Heidelberg: Winter.

Brehm, Reinhold und Theodor F. Zimmermann 1865. Bilder und Skizzen aus dem Zoologischen Garten zu Hamburg. Hamburg: Lührsen.

Brönnle, K. W. 1910. Ein deutscher Tiermaler. Zum Gedächtnis Friedrichs Spechts. Reclams Universum (26): 32-39.

Brower, Matthew 2010. Developing Animals. Wildlife and Early American Photography. Minneapolis, London: University of Minnesota Press.

Cahn, Paul 1906. H. Meerwarth, Photographischen Naturstudien. Eine Anleitung für Amateure und Naturfreunde. Verlag v. J.F. Schreiber. Zoologischer Beobachter/Der Zoologische Garten (47): 158-159.

Chadarevian, Soraya de 1994. Sehen und Aufzeichnen in der Botanik des 19. Jahrhunderts. In: Michael Wetzel und Herta Wolf (Hg.). Der Entzug der Bilder. München: Fink: $121-144$. 
Clegg, Brian 2007. The Man who Stopped Time. The Illuminating Story of Eadweard Muybridge: Father of the Motion Picture, Pioneer of Photography, and Murderer. Washington D.C.: Henry Press.

Cornish, Charles J. 1896. Life at the Zoo. Notes and Traditions of the Regent's Park Gardens. 4. Aufl. London: Seeley.

Daston, Lorraine und Peter Galison 2007. Objektivität. Frankfurt/M.: Suhrkamp.

Daum, Andreas W. 2002. Wissenschaftspopularisierung im 19. Jahrhundert. Bürgerliche Kultur, naturwissenschaftliche Bildung und die deutsche Öffentlichkeit, 1848-1914. 2. Aufl. München: Oldenbourg.

Deimann, Wilhelm 1927. Vom Werden der Lönschen Tierdichtung. (An der Hand von Lönsbriefen). Markwart. Mitteilungen der Deutschen Hermann-Löns-Gesellschaft (3), 8: 113-119.

Dunaway, Finis 2000. Hunting with the Camera: Nature Photography, Manliness, and Modern Memory, 1890-1930. Journal of American Studies (34): 207-230.

Dupke, Thomas 1994. Hermann Löns. Mythos und Wirklichkeit. 2. Aufl. Hildesheim: Claassen.

Engels, Eve-Marie 1995. Biologische Ideen von Evolution im 19. Jahrhundert und ihre Leitfunktionen. Eine Einleitung. In: Eve-Marie Engels (Hg.). Die Rezeption von Evolutionstheorien im 19. Jahrhundert. Frankfurt/M.: Suhrkamp: 13-66.

Ettlinger, Max 1910. Die Wiederbelebung biologischer Beobachtungsfreude. Hochland (7), 5: 619-622.

Faulstich, Werner 2004. Medienwandel im Industrie- und Massenzeitalter (1830-1900). Göttingen: Vandenhoeck \& Ruprecht (= Die Geschichte der Medien 5).

Finsch, Otto 1868. Dr. Brehm's Thierleben. „Abtheilung Vögel“. Hildburghausen, Verlag des Bibliographischen Instituts. 40 Hefte. Journal of Ornithology (16): 58-65.

Gall, Alexander 2011. Authentizität, Dramatik und der Erfolg der populären zoologischen Illustration im 19. Jahrhundert: Brehms Thierleben und die Gartenlaube. In: Stefanie Samida (Hg.). Inszenierte Wissenschaft. Zur Popularisierung von Wissen im 19. Jahrhundert. Bielefeld: transcript: 103-126.

Gall, Alexander 2016. Auf dem langen Weg ins Museum. Dioramen als kommerzielle Spektakel und Medien der Wissensvermittlung im langen 19. Jahrhundert. In: Alexander Gall und Helmuth Trischler (Hg.). Szenerien und Illusionen. Geschichte, Varianten und Potenziale von Museumsdioramen. Göttingen: Wallstein: 27-106.

Gebauer, Mirjam 2013. „Quellen ästhetischen Genusses und veredelnder Erkenntnis“. Zur Naturauffassung in Ernst Haeckels Kunstformen der Natur. In: Adam Paulsen und Anna Sandberg (Hg.). Natur und Moderne um 1900. Räume - Repräsentationen - Medien. Bielefeld: transcript: 247-264.

Giebel, Christoph G. 1859. Die Säugethiere (Mit 926 Abbildungen). In: Christoph G. Giebel, Die Naturgeschichte des Thierreichs, Bd. 1. Leipzig: Wigand (= Die Drei Reiche der Natur. Erste Abtheilung).

Gissibl, Bernhard 2005. Exotische „Natururkunden“. Tierfotografie im Kontext des deutschen Kolonialismus. In: Ute Eskildsen und Hans-Jürgen Lechtreck (Hg.). Nützlich, süß und museal: Das fotografierte Tier. Essays. Göttingen: Museum Folkwang, Steidl: 60-69.

Graf, Andreas und Susanne Pellatz 2003. Familien- und Unterhaltungszeitschriften. In: Georg Jäger (Hg.). Geschichte des deutschen Buchhandels im 19. und 20. Jahrhundert. Das Kaiserreich 1871-1918, Teil 2. Frankfurt/M.: Buchhändler-Vereinigung: 409-522.

Guggisberg, Charles A. 1977. Early Wildlife Photographers. Foreword by Eric Hosking. Newton Abbot, London, Vancouver: David \& Charles.

Haemmerlein, Hans-Dietrich 1989. Brehms Tierleben - ein vielschichtiges Erbe. BrehmBlätter (3): 13-29.

Haemmerlein, Hans-Dietrich 1993. Dokumentarisches zur Afrikareise des Herzogs Ernst II., 1862. In: Harald Bachmann u. a. (Hg.). Herzog Ernst II. von Sachsen-Coburg und Gotha, 1818-1893 und seine Zeit. Jubiläumsschrift im Auftrag der Städte Coburg und Gotha. Augsburg: Maro: 419-451.

Haemmerlein, Hans-Dietrich 2015. Alfred Edmund Brehm. Biografie in Zeit- und Selbstzeugnissen. Markkleeberg: Sax. 
Haffer, Jürgen 2001. Ornithological Research Traditions in Central Europe During the 19th and 20th centuries. Journal of Ornithology (142), Supplement 1: 27-93.

Hanstein, Reinhold von 1906. Besprechungen. Kiessling, M., Anleitung zum Photographieren freilebender Tiere; Meerwarth, H., Photographische Naturstudien; Kearton, R., Tierleben in freier Natur. Natur und Schule (5): 323-326.

Hauffe, Friederike und Heinz-Georg Klös 1995. Der Tierillustrator Gustav Mützel. Bongo (26): 29-46.

Heck, Ludwig 1899. Lebende Bilder aus dem Reiche der Tiere. Augenblicksaufnahmen nach dem lebenden Tierbestande des Berliner Zoologischen Gartens. Berlin: Werner.

Heck, Ludwig 1905. C. G. Schillings und sein Erstlingswerk. Ein Vorwort. In: Carl G. Schillings, Mit Blitzlicht und Büchse. Neue Beobachtungen und Erlebnisse in der Wildnis inmitten der Tierwelt von Äquatorial-Ostafrika. Leipzig: Voigtländer: 1-6.

Heck, Ludwig 1938. Heiter-ernste Lebensbeichte. Erinnerungen eines alten Tiergärtners. Berlin: Globus.

Heinroth, Oskar 1905. Buchbesprechung: C.G. Schillings, Mit Blitzlicht und Büchse. Naturwissenschaftliche Rundschau (20 = N.F. 4), 4: 60-62.

Herrmann, Anja 2014. Imaginäre Flanerien. Das Fotoatelier als Ort der Moderne. Rundbrief Fotografie (21), 3: 27-35.

Hochadel, Oliver 2011. Watching Exotic Animals Next Door: "Scientific" Observations at the Zoo (ca. 1870-1910). Science in Context (24): 183-214.

Hopwood, Nick 2005. Visual Standards and Disciplinary Change. Normal Plates, Tables and Stages in Embryology. History of Science (43): 239-303.

Jäger, Georg 2001. Der Lexikonverlag. In: Georg Jäger (Hg.). Geschichte des deutschen Buchhandels im 19. und 20. Jahrhundert. Das Kaiserreich 1871-1918, Teil 1. Frankfurt/M.: Buchhändler-Vereinigung: 541-574.

Janecke, Christian 2010. „Inszenierte Fotografie“, „Inszenierende Fotografie“ und „Fotografierte Inszenierung" - am Beispiel von Schauanordnungen für lebende und tote Tiere. In: Lars Blunck (Hg.). Die fotografische Wirklichkeit. Inszenierung - Fiktion - Narration. Bielefeld: transcript: 53-70.

Kaufhold, Enno 1986. Bilder des Übergangs. Zur Mediengeschichte von Fotografie und Malerei in Deutschland um 1900. Marburg: Jonas.

Kelly, Alfred 1981. The Descent of Darwin. The Popularization of Darwinism in Germany, 1860-1914. Chapel Hill: University of North Carolina Press.

Kiesling, Martin 1901. Photographieen lebender Tiere. Photographische Mitteilungen (38), 2: $35-41$.

Kiesling, Martin 1905. Anleitung zum Photographieren freilebender Tiere. Mit einem Anhang von Dr. A. Voigt. Leipzig: Voigtländer.

Knoepfelmacher, U. C. und G. B. Tennyson 1977. Introduction. In: U. C. Knoepfelmacher and G. B. Tennyson (Hg.). Nature and the Victorian Imagination. Berkeley: University of California Press: xvii-xxiii.

Kockerbeck, Christoph 1986. Ernst Haeckels „Kunstformen der Natur“ und ihr Einfluß auf die deutsche bildende Kunst der Jahrhundertwende. Studie zum Verhältnis von Kunst und Naturwissenschaften im Wilhelminischen Zeitalter. Frankfurt/M.: Lang.

Köstering, Susanne 2003. Natur zum Anschauen. Das Naturkundemuseum des deutschen Kaiserreichs 1871-1914. Wien: Böhlau.

Lechtreck, Hans-Jürgen 2005. Fotografie und Tierpräparation komplementär. Anmerkungen zur gemeinsamen Geschichte zweier zoologischer Aufzeichnungsmedien des 19. Jahrhunderts. In: Ute Eskildsen und Hans-Jürgen Lechtreck (Hg.). Nützlich, süß und museal: Das fotografierte Tier. Essays. Göttingen: Museum Folkwang, Steidl: 70-95.

Lightman, Bernard V. 2007. Victorian Popularizers of Science. Designing Nature for New Audiences. Chicago: University of Chicago Press.

Löns, Hermann 1905. Aufruf! Die Wirbeltierfauna der Provinz Hannover. Der Zoologische Garten (46): 124.

Ludwig, Heidrun 2000. Joseph Wolf in Darmstadt (1841-1847) - Zur Bedeutung seines Frühwerks. In: Karl Schulze-Hagen und Armin Geus (Hg.). Joseph Wolf (1820-1899), Tiermaler. Marburg/Lahn: Basilisken-Presse: 69-114.

Meerwarth, Hermann 1905. Photographische Naturstudien. Eine Anleitung für Amateure und Naturfreunde. Eßlingen, München: Schreiber. 
Meerwarth, Hermann 1909 [1908]. Vorwort. In: Hermann Meerwarth (Hg.). Lebensbilder aus der Tierwelt. Erster Band. Erste Folge: Säugetiere I. 2. Aufl. Leipzig: Voigtländer: V-VIII.

Meerwarth, Hermann (Hg.) 1908. Lebensbilder aus der Tierwelt. Sonderheft: Das Tierbild der Zukunft. Leipzig: Voigtländer.

Mey, Eberhard 1989. Über die Illustrationen in „Brehms Thierleben“. [Unveröffentlichter Vortrag auf dem Kolloquium der Brehm Gedenkstätte Renthendorf am 11. Nov.].

Millard, Charles 1977. Images of Nature: A Photo-Essay. In: U. C. Knoepfelmacher and G. B. Tennyson (Hg.). Nature and the Victorian Imagination. Berkeley: University of California Press: 3-26.

Müllenhoff, Karl 1885/1886. Die Momentphotographie im Dienste naturwissenschaftlicher Forschungen. Westermanns illustrierte deutsche Monatshefte (59): 388-399.

Müller, Conrad 1887. Die Augenblicksphotographie. („Der Reichsbote“ Februar 1887). In: Ottomar Anschütz (Hg.). Die Augenblicksphotographie. Ihr Wesen, ihre Bedeutung, ihre Ziele dargestellt in Aufsätzen. Lissa (Selbstverlag) 67-85.

Müller-Liebenwalde, Johannes 1893. Gustav Mützel. Eine biographische Skizze (Nachruf). Der Zoologische Garten (34): 321-328.

Nickelsen, Kärin 2007. „Abbildungen belehren, entscheiden Zweifel und gewähren Gewissheit" - Funktionen botanischer Abbildungen im 18. Jahrhundert. Wiener Zeitschrift zur Geschichte der Neuzeit (7): 52-70.

Noll, Friedrich C. 1884. [Besprechung: ] Die Säugetiere in Wort und Bild, von Carl Vogt und F. Specht. Der Zoologische Garten (25): 29-32.

Noll, Friedrich C. 1889. Photographie und Zoologie. Der Zoologische Garten (30): 135-139.

Nyhart, Lynn K. 2009. Modern Nature. The Rise of the Biological Perspective in Germany. Chicago: University of Chicago Press.

Pechuël-Loesche, Eduard 1890. Die Säugetiere. In: Eduard Pechuël-Loesche (Hg.). Brehms Tierleben. Allgemeine Kunde des Tierreichs, Bd. 1. 3. Aufl. Leipzig, Wien: Bibliogr. Inst.

Peters, Dorothea 2004. Vom gedruckten Foto zur Luxuskleinkunst. Die Bildproduktion der Graphischen Kunstanstalt Meisenbach, Riffarth \& Co. Gutenberg-Jahrbuch (79): 219-250.

Pohlmann, Ulrich 2004a. Vera Icon oder Die Wahrheit der Fotografie. In: Ulrich Pohlmann und Johann G. von Hohenzollern (Hg.). Eine neue Kunst? Eine andere Natur! Fotografie und Malerei im 19. Jahrhundert. München: Schirmer/Mosel: 9-14.

Pohlmann, Ulrich 2004b. Tierstudien. In: Ulrich Pohlmann und Johann G. von Hohenzollern (Hg.). Eine neue Kunst? Eine andere Natur! Fotografie und Malerei im 19. Jahrhundert. München: Schirmer/Mosel: 98-115.

Pöppig, Eduard 1851. Naturgeschichte der Säugetiere. Mit 1100 Abbildungen. In: Eduard Pöppig, Illustrirte Naturgeschichte des Thierreichs. Anatomie, Physiologie und Geschichte der Säugethiere, der Vögel, der Lurche, der Fische und der wirbellosen Thiere, $B d$. 1. Leipzig: Weber.

R. Voigtländers Verlag 1905. Aufruf an alle europäischen Berufs- und Liebhaber-Photographen! In: Martin Kiesling, Anleitung zum Photographieren freilebender Tiere. Leipzig: Voigtländer: 83-86.

Rieke-Müller, Annelore 1995. Angewandte Zoologie und die Wahrnehmung exotischer Natur in der zweiten Hälfte des 18. und im 19. Jahrhundert. History and Philosophy of the Life Sciences (17): 461-484.

Rieke-Müller, Annelore und Lothar Dittrich 1998. Der Löwe brüllt nebenan. Die Gründung Zoologischer Gärten im deutschsprachigen Raum 1833-1869. Köln: Böhlau.

Rossell, Deac 2001. Faszination der Bewegung. Ottomar Anschütz zwischen Photographie und Kino. Basel, Frankfurt/M.: Stroemfeld.

Roßmäßler, Emil A. 1862. Des Herzogs Ernst Reise nach dem tropischen Afrika. Aus der Heimath (4), 7: 97-100.

Ryan, James R. 1997. Picturing Empire. Photography and the Visualization of the British Empire. London: Reaktion Books.

Sarkowski, Heinz 1976. Das Bibliographische Institut. Verlagsgeschichte und Bibliographie 1826-1976. Mannheim: Bibliograph. Inst.

Schillings, Carl G. 1905. Mit Blitzlicht und Büchse. Neue Beobachtungen und Erlebnisse in der Wildnis inmitten der Tierwelt von Äquatorial-Ostafrika. Mit 302 urkundtreu in 
Autotypie wiedergegebenen photographischen Original-Tag-und Nacht-Aufnahmen des Verfassers. 2. Aufl. Leipzig: Voigtländer.

Schillings, Carl G. 1906. Der Zauber des Elelescho. Mit 318 Abbildungen, meist photographischen Original-Tag- und Nachtaufnahmen des Verfassers, urkundtreu in Autotypie wiedergegeben. Leipzig: Voigtländer.

Schirrmacher, Arne 2008. Nach der Popularisierung. Zur Relation von Wissenschaft und Öffentlichkeit im 20. Jahrhundert. Geschichte und Gesellschaft (34): 73-95.

Schlawe, Lothar 1994. Illustrationen nach dem Leben (ndL) aus dem Zoologischen Garten zu Berlin. Bongo (23): 35-62.

Schneider, Bernhard 1988a. Drei Briefe von A. E. Brehm an Carl Bolle im Zusammenhang mit dem Entstehen des „Thierlebens“, geschrieben im Jahre 1861 von Leipzig aus. Veröfentlichungen Naturkundemuseum Leipzig (5): 51-64.

Schneider, Bernhard 1988b. Leipzig - ein Boden für eine Pflanze wie Alfred. Der Leipziger Aufenthalt (1858-1862) von Alfred Edmund Brehm und seine Freundschaft mit dem Naturforscher und naturwissenschaftlichen Volkslehrer Emil Adolf Roßmäßler. Leipzig. Aus Vergangenheit und Gegenwart. Beiträge zur Stadtgeschichte (5): 29-67.

Schneider, Bernhard 1991. Aus dem Briefwechsel von Dr. A. E. Brehm und Herrmann Meyer, Geschäftsführer des Bibliographischen Instituts, im Zusammenhang mit der Herausgabe des „Thierlebens“. Veröffentlichungen Naturkundemuseum Leipzig (9): 55-76.

Schöberl, Joachim 1996. „Verzierende und erklärende Abbildungen“. Wort und Bild in der illustrierten Familienzeitschrift des neunzehnten Jahrhunderts am Beispiel der Gartenlaube. In: Harro Segeberg (Hg.). Die Mobilisierung des Sehens. Zur Vor- und Frühgeschichte des Films in Literatur und Kunst. München: Fink: 209-236.

Schulze, Andreas 2009. „Belehrung und Unterhaltung“. Brehms Tierleben im Spannungsfeld von Empirie und Fiktion. München: Utz.

Schulze, Elke 2005. Zeichnung und Fotografie - Statusfragen. Universitäres Zeichnen und naturwissenschaftliche Bildfindung. Berichte zur Wissenschaftsgeschichte (28): 151-159.

Schulz, Georg E. 1908/1909. Natur-Urkunden. Biologisch erläuterte photographische Aufnahmen frei lebender Tiere und Pflanzen. 8 Bde., Berlin: Parey.

Schulz, Georg E. 1908. Natur-Urkunden. Biologisch erläuterte photographische Aufnahmen frei lebender Tiere und Pflanzen. Heft 1 Vögel. Berlin: Parey (= Erste Reihe).

Schütt, F. 1895. Vereinsnachrichten. Freie photographische Vereinigung zu Berlin. Sitzung vom 15. Februar 1895. Photographische Rundschau (9), 4:1-2.

Schwarz, Angela 1999. Der Schlüssel zur modernen Welt. Wissenschaftspopularisierung in Großbitannien und Deutschland im Übergang zur Moderne (ca. 1870-1914). Stuttgart: Steiner.

Schwarz, Angela 2002. Populärwissenschaftlich in Text und Bild? Zur Visualisierung in der britischen Populärwissenschaft des 19. Jahrhunderts: Das Beispiel der Literatur für Kinder und Jugendliche. Archiv für Geschichte des Buchwesens (56): 179-202.

Schwarz, Angela 2007. Die Popularität der Popularisierung: Allgemeinverständliche Bücher über Naturwissenschaften und der Anteil der Verleger an ihrem Erfolg im 19. Jahrhundert. In: Monika Estermann und Ute Schneider (Hg.). Wissenschaftsverlage zwischen Professionalisierung und Popularisierung. Wiesbaden: Harrassowitz: 61-76.

Staab, Joachim F. 1990. Nachrichtenwert-Theorie. Formale Struktur und empirischer Gehalt. Freiburg/Br., München: Alber.

Storsberg, Klaus 2010. Naturfotografie 1839-1964 in Deutschland. Ein historischer Rückblick. Leverkusen: Digital Imaging-Nature-Verl.

Urania Berlin 1913. Denkschrift zum 25jährigen Bestehen der Gesellschaft Urania in Berlin (1888-1913). Berlin: Büxenstein.

Virchow, Rudolf 1890. Brehm's Tierleben. Dritte, gänzlich neu bearbeitete Auflage von Prof. Dr. Pechuël-Loesche. Säugetiere. 1. Bd. Zeitschrift für Ethnologie (22): 170 -171.

Vogt, Hermann 1886. Etwas über Momentphotographie. Vom Fels zum Meer (11): 339-346.

Voss, Julia 2005. Zoologische Gärten, Tiermaler und die Wissenschaft vom Tier im 19. Jahrhundert. In: Christiane Groeben, Joachim Kaasch und Michael Kaasch (Hg.). Stätten biologischer Forschung | Places of biological research. Beiträge zur 12. Jahrestagung der DGGTB in Neapel 2003. Berlin: VWB: 227-243. 
Voss, Julia 2007. Darwins Bilder. Ansichten der Evolutionstheorie 1837 bis 1874. Frankfurt/M.: Fischer.

Voss, Julia 2009. Variieren und Selektieren: Die Evolutionstheorie in der englischen und deutschen illustrierten Presse im 19. Jahrhundert. In: Pamela Kort und Max Hollein (Hg.). Darwin. Kunst und die Suche nach den Ursprüngen. Köln: Wienand: 246-257.

Voss, Sonja 2015. George Shiras and the Birth of Wildlife Photography. In: Jean-Christophe Bailly und Sonja Voss (Hg.). George Shiras: In the Heart of the Dark Night. Paris: Éditions Xavier Barral: 81-89.

Wandolleck, Benno 1911. Zoologie und Physiologie. In: Karl W. Wolf-Czapek (Hg.). Angewandte Photographie in Wissenschaft und Technik in vier Teilen, Bd. 2. Berlin: Union Dt. Verl.-Ges.: 37-66.

Weinland, David F. 1860a. Einige Gedanken über die Thierseele. Der Zoologische Garten (1): 129-134.

Weinland, David F. 1860b. Was zu einem "ganzen“ Thiere gehört und wie man vielleicht Tropenthiere gesünder erhalten könnte. Der Zoologische Garten (1): 185-187.

Weinland, David F. 1862. Thierzeichnungen. Der Zoologische Garten (3): 20.

Weinland, David F. 1863. Löwenstudien. Der Zoologische Garten (4): 30-32.

Weise, Bernd 2003. Aktuelle Nachrichtenbilder „nach Photographien“ in der deutschen illustrierten Presse der zweiten Hälfte des 19. Jahrhunderts. In: Charles Grivel, André Gunthert und Bernd Stiegler (Hg.). Die Eroberung der Bilder. Photographie in Buch und Presse (1816-1914). München: Fink: 62-101.

Wildmeister, Birgit 1998. Die Bilderwelt der „Gartenlaube“. Ein Beitrag zur Kulturgeschichte des bürgerlichen Lebens in der zweiten Hälfte des 19. Jahrhunderts. Würzburg: Bayerische Blätter für Volkskunde.

Wilke, Jürgen 1984. Nachrichtenauswahl und Medienrealität in vier Jahrhunderten. Eine Modellstudie zur Verbindung von historischer und empirischer Publizistikwissenschaft. Berlin: de Gruyter.

Wittmann, Barbara 2008. Das Portrait der Spezies. Zeichnen im Naturkundemuseum. In: Christoph Hoffmann (Hg.). Daten sichern. Schreiben und Zeichnen als Verfahren der Aufzeichnung. Zürich: Diaphanes: 47-72.

Wood, John G. 1859. The Illustrated Natural History. Vol. I. Mammalia. London: Routledge.

Wurm, Wilhelm 1897. Naturgeschichte und Charakterschilderung der zur hohen Jagd gehörigen Thiere Mitteleuropas. Ein Beitrag zur wissenschaftlichen und zur praktischen Thierkunde. Leipzig: Vogel.

\author{
Alexander Gall \\ Forschungsinstitut für Technik- und Wissenschaftsgeschichte \\ Deutsches Museum \\ 80306 München \\ Deutschland \\ a.gall@deutsches-museum.de
}

